\title{
Aerosol Dynamics in a sputtering DC discharge
}

\author{
A. Michau ${ }^{a, 1}$, C. Arnas ${ }^{b}, K$. Hassouni $i^{a, 1}$ \\ ${ }^{a}$ LSPM, CNRS, 99 Avenue J. B. Clément, 93430 Villetaneuse, France \\ ${ }^{b}$ CNRS, Aix-Marseille université, LaboratoirePIIM, Centre Saint-Jérôme, 13397 Marseille, France
}

\section{Abstract:}

An aerosol sectional model that describes the space-time evolution of the size distribution of carbon particles produced in a graphite cathode sputtering discharge is presented. The model includes the coupled phenomena of particle growth processes, due to coagulation and molecular surface deposition, particle nucleation as inferred from a molecular growth process, particle charging in the plasma and particle drift-diffusion transport in the electrode gap. Comparison between simulation results and experiments showed that the model yields a satisfactory prediction of the particle cloud density and particle mean diameter in the electrode gap. It also yields a satisfactory prediction of the particle size distribution. The calculated size distribution shows two particle-populations: a first one with a very small size and just above the nucleus diameter and a second population, with a larger size, that determines the mean-diameter of the particle cloud. The sectional model also shows that simple aerosol models assuming single size particles significantly overestimates the particle density although it yields a satisfactory prediction for the particle size.

Key-words : DC discharges, dusty plasma, aerosol dynamic, cluster growth, model

${ }^{1}$ corresponding author : armelle.michau@lspm.cnrs.fr, khaled.hassouni@lspm.crns.fr 
This manuscript was accepted by J. Appl. Phys. Click here to see the version of record.

\section{Introduction}

Although solid particle formation is observed in low pressure DC discharges since the 90's [1], [2], very little effort has been devoted to the understanding of the processes that lead to their formation. Recently a first modeling study of dusty plasmas generated in graphite cathode argon DC discharges showed that particle formation is induced by molecular growth and a subsequent electron attachment on carbon clusters [3]. Attachment results in the formation of negatively charged clusters that are trapped in the field reversal region of the negative glow (NG) and can therefore undergo further growth that end up with solid particle nucleation. In the model used in this first investigation, the coupling between the discharge equilibrium and the particle aerosol dynamic was not taken into account. Nevertheless, it was clearly shown that the field reversal effect in the NG plays a key-role as far as particle nucleation in DC sputtering discharges is concerned [3]. Based on this first approach, an improved self consistent dusty plasma model was developed. This model couples the discharge equilibrium, the molecular growth kinetic and the particle aerosol dynamic described through the number-density, the mean-diameter and the average-charge of the particles. This second study showed that the field reversal effect still enables particle formation when the coupling between the space charge field and particle formation is taken into account [4]. It also showed that this coupling effect is effective only for long discharge durations, i.e., longer than $300 \mathrm{~s}$ duration.

In all these studies, the dust particle clouds were described using their total particle densities and mean-diameters. A major consequence of such a simplification is that coagulation kinetic is not accurately described. As a matter of fact, dust particles are charged in plasma discharges, and assuming that all the particles have a size corresponding to the meandiameter results in a particle with a substantial negative charge (this is a consequence of the Orbit Motion Limited (OML) charge equilibrium). Consequently, with this simple particle cloud description, the charge fluctuation, which is the driver for particle coagulation, is not high enough to generate neutral or positively charged particles and the model tends to artificially eliminate the coagulation processes [4]. Consequently, accurate prediction of the coagulation kinetics requires taking into account the detailed particle size distribution so as to access the fraction of small size particles that may be either neutral or positively charged due to the charge fluctuation effect.

Therefore, in this paper, we are interested in investigating the detailed size distribution over the whole particle population through an aerosol dynamics approach. The space-time evolution of the particle size distribution is governed by the interplay between the nucleation, the growth through cluster deposition on the particle surface and the coagulation [5],[6]. The kinetics of these phenomena are highly dependent on the electrical charge and on the surface to volume ratio of the particles. For this purpose we will make use of the so-called sectional model that enables one considering the size distribution over a large range of particle size. A size range of 1-100 nm was considered in this study, which allows obtaining a detailed description of the governing growth mechanisms as function of 
This manuscript was accepted by J. Appl. Phys. Click here to see the version of record.

the discharge duration, the position in the discharge gap and the particles size and charge. In order to simplify the analysis and keep the numerical solution tractable, we do not consider in this study the coupling between charged carbon particles and discharge equilibrium. We limit ourselves to discharge durations where this coupling can be neglected.

The paper is organized in five sections, including this introduction. In section 2, the investigated discharge is briefly presented. The model used to describe the investigated dusty plasmas is described in section 3 . In section 4 , we discuss the results obtained on the time-space evolution of the particle cloud characteristics, i.e. size-distribution, particle density, average diameter, charge, etc. The calculated results are compared with experimental measurements. We also discuss how the interplay between the different phenomena involved in the aerosol dynamic end up with the observed particle size distributions. The main conclusions that may be drawn from this work are given in section 5 .

\section{Model Description}

\section{II.1. Investigated discharge system}

We consider an argon DC glow discharge, produced between two parallel plane electrodes of $10 \mathrm{~cm}$ diameter [7]. The cathode is in graphite and the grounded anode in stainless steel. The distance between the electrodes is $14 \mathrm{~cm}$. The argon pressure in the vessel is $60 \mathrm{~Pa}$ and the discharge current is adjusted to a constant value of $80 \mathrm{~mA}$. This results in a discharge voltage of $550 \mathrm{~V}$ and since the anode is grounded, the cathode takes spontaneously a bias value of approximately $-550 \mathrm{~V}$. As a consequence, the cathode is sputtered by ions and by fast argon atoms that are produced through charge exchange in the cathode sheath [8]. The sputtering of the graphite cathode releases in the emission of $\mathrm{C}$-atom and, to a lesser extent $C_{2}$ and $C_{3}$ clusters, in the gas phase [9]. Clusters are transported in the discharge where they experience cluster-cluster aggregation and electron attachment processes. Once negative clusters are formed they are trapped in the field reversal region of the DC potential structure [3]. Due to this trapping effect, they have high enough residence time so that they can grow until they reach a critical size at which nucleation occurs [3].

\section{I. 2. Model characteristics and modeling approach}

The molecular growth pathway leading to particle nucleation was extensively studied and discussed in [10], [3], [11]. We are here interested in the behavior of the particle in the discharge once they nucleate. More specifically we seek information on the time and space evolution of the particle size distribution. As a matter of fact, particles, once formed, can grow by two mechanisms, i.e., molecular deposition on their surface and particle-particle coagulation. The relative predominance of these mechanisms strongly depends of the particle size and charge distributions.

As far as coagulation process is concerned, collision probability between two charged particles strongly depends on the electrostatic attraction/repulsion effect. This is governed 
by the instantaneous charge-values of the colliding particles. The charge value of a given particle shows a significant dynamic fluctuation due to the stochastic nature of electrons and ions collection by the solid particles in the plasma. Under the studied discharge conditions, in particular with an electron temperature of $0.1 \mathrm{eV}$, the typical elementary charge, as may be inferred from the OML theory for particle size is in the range 1-100 $\mathrm{nm}$, are relatively small, i.e., between -1 and -20 elementary negative charges [12]. Note that these values are similar to those reported by Agarwal and al. [13] and Kim et al. [14] for radiofrequency dusty silane plasmas. In these conditions, the charge fluctuation can lead to the existence of particles carrying instantaneous neutral and even positive charge. We follow the work of Matsukas et al. [15] and made the assumption that the particle charge is given by a Gaussian distribution around an average charge for a given particle diameter. Note that even if the assumptions made in Matsoukas et al [15], are not totally fulfilled for the smallest particle, we use this assumption for all particle size distribution. This allows us to account, in a simple way, for the particle charge distribution that enables charged particle coagulation. It is worthy to mention here, that coagulation may be also enhanced through a dipole interaction between particle [16]. This mechanism was however not taken into account in this study.

The values of the colliding particle diameters are also key-factors that affects the coagulation frequency. Figure 1 depicts the variation of the normalized coagulation frequency with the diameter ratio of the colliding particles. This evolution shows that two particles with very different diameters have much higher coagulation frequencies than particles with the same size. As an example, the probability value for the coagulation between 1 and $100 \mathrm{~nm}$ particles is three orders of magnitude larger than that for two $100 \mathrm{~nm}$-diameter particles.

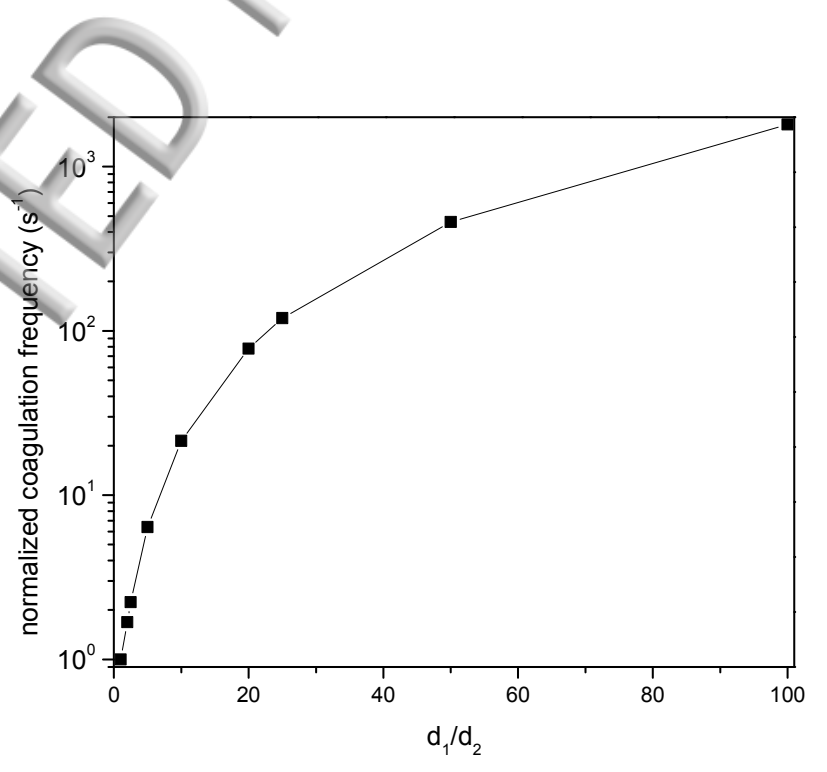

Figure 1 : Normalized coagulation frequency between two neutral particles of diameter $d_{1}$ and $d_{2}$ from [5] 
Therefore, a satisfactory description of the particle coagulation process requires taking into account the whole particle size distribution. And, indeed, a model, denoted in the following a single size model, that assumes that all the particle have the same size, corresponding to the mean diameter, would significantly underestimate the coagulation contribution to the overall aerosol dynamics [3].

We propose in this study to analyze the dust growth and transport phenomena using the socalled sectional model where particle size range is divided into bins, or sections, which allows a detailed description of the particle size effect.

Aerosol dynamics in a DC discharge strongly depend on the discharge conditions. This means that describing particle nucleation and growth resulting from molecular sputtering of small precursors in the discharge volume should take into account the coupling between the plasma equilibrium, the molecular species balances and the aerosol dynamics. Such a coupling that governs the dusty plasma effect was previously investigated using a single size aerosol model where the particle charge and size distributions are described by average values [11]. Taking this coupling into account in the frame of a detailed study of aerosol dynamics using a sectional model turns out to be computationally cumbersome.

Since we are mainly interested in the detailed evolution of the particle size distribution, we will consider a discharge durations shorter than $300 \mathrm{~s}$ for which the coupling between particle dynamics and discharge equilibrium is almost negligible [11]. For such discharges, the aerosol dynamics may be well described without taking into account the dusty plasma effect (the contribution of the particles in the plasma equilibrium may be neglected). Further, the aerosol dynamics is coupled to the cluster kinetics through particle nucleation and cluster loss due to their sticking on the particle surface. We showed that the cluster deposition is predominant for species $C_{1} C_{2}$ and $C_{3}$ which are produced from the graphite cathode sputtering [3]. These densities are controlled mainly by the balance between their production through sputtering, and their consumption due to aggregation and sticking. Taking into account the full coupling between cluster kinetics and a detailed aerosol dynamics also turns out to be very cumbersome. We assumed therefore in this work that the space-time variation of the cluster densities and nucleation rate are similar to those obtained with the model that describes the particle cloud using a single size distribution, i.e., corresponding to the mean-diameter and average charge.

Consequently, the investigation of the detailed aerosol dynamic was performed using a twostep approach. In a first step, we solve for the plasma equilibrium together with a cluster module and a single-size particle distribution model [3]. This first step allows in particular determining the time and space evolution of the plasma parameters, i.e., electron density and electric field, and the molecular cluster densities.

In a second step, we solve for the detailed aerosol dynamics using the time and space distributions of the plasma parameters and cluster densities calculated in the first step. Our 
approach assumes therefore that the fully coupled model using a single size particle distribution corresponding to averaged particle properties yields satisfactory estimates of the cluster densities that govern the particle nucleation and growth through surface deposition processes.

In the next sections we briefly present the models used to describe the plasma equilibrium, the molecular growth and the aerosol dynamic.

\section{II.3. Plasma module}

We restrict ourselves to the description of the main peculiarities of this model that was thoroughly discussed in [3]. As mentioned previously we are interested in discharge durations for which the plasma equilibrium is not affected by the particles and negative clusters. Therefore the DC discharges characteristics were determined assuming a pristine argon discharge and disregarding the effect of carbon species in the charge balance.

We used a 1D model that describes the discharge characteristics across the electrode gap. We distinguish three discharge regions: the cathode sheath, the negative glow (NG) and the Faraday dark space (FDS). The situation considered here corresponds therefore to a short DC discharge without a positive column region. A non-local approach proposed by Kolobov and Tsendin [17] is combined to a Monte-Carlo simulation of the high-energy electrons to determine the sheath dimension and the ionization rate along the discharge gap. We then solve for the cold electron density profile with the assumption of an ambipolar transport using the ionization rate inferred from Monte Carlo simulations in both the NG and the FDS. We made the assumption that the cold electron temperature is $0.1 \mathrm{eV}$ [17] . The electric field is finally determined assuming that cold electrons are in Boltzmann equilibrium[18].

\section{II.4. Cluster module}

For the applied voltage value of $-600 \mathrm{~V}$ considered in this work, only small clusters $C_{1} C_{2}$ and $\mathrm{C}_{3}$ can be sputtered from the graphite cathode [9] . These primary clusters have to grow until nucleation can occur. For the discharge pressure considered here, diffusion is fast and neutral clusters are lost on the reactor wall before undergoing any molecular growth [4]. Similarly, positive carbon clusters that may form by electron impact ionization or charge transfer processes are rapidly lost by drift to the electrode in the high electric field cathode sheath and radial diffusion to the reactor wall outside the sheath [11]. In fact, for the conditions considered here, only negative clusters obtained by attachment processes and trapped in the field reversal region can show high enough residence time to enable particles nucleation [3]. Consequently, the molecular growth model used to determine the nucleation rate takes into account negative and neutral clusters and disregards positively charged clusters. Carbon clusters are subject to aggregation, attachment and detachment processes discussed in [3]. Particle nucleation is assumed to originate from the aggregation processes 
involving a largest molecular edifice (LME). Although, the size of this later is very difficult to assess, we showed in a previous study that LME above 15 carbon atoms results in similar nucleation rates [19]. We therefore assume in this work that the nucleation proceeds through the aggregation processes involving $C_{15}$ and $C_{15}{ }^{-}$.

\section{II.5. Aerosol dynamics model}

The detailed charge and size distributions can be well described using sectional models [20],[21] that make possible taking into account the size and charge effects in nucleation, coagulation and surface growth by cluster sticking processes. Sectional models were especially used for modelling the aerosol dynamics in dusty silane plasmas obtained in capacitively coupled radiofrequency discharges (CCRF) [22],[23], [24],[25],[26],[16],[13]

In this work, we develop a 1D sectional model to describe carbon nanoparticle nucleation, growth and transport in DC argon discharge. In our approach, the space and time-variation of particle nucleation rate is inferred from the cluster growth model briefly presented in the previous section and thoroughly described in reference [3]. The same cluster molecular growth model is used to determine the cluster densities required for the estimation of particle growth kinetics through surface sticking processes. Particle charge distribution is included in this model considering a Gaussian distribution around a dynamic average charge for each section of the size distribution. The average charge for each section is calculated using charge balance equations [4].

We present in this section a brief description of this sectional model where the size distribution is investigated over a finite size range that is divided into $n$ evenly spaced sections of particle volume logarithm $z: z=\ln (v)$. Particles of section $i$ have volumes in the range $\left[v_{i}, v_{i+1}\right]$ such as $v_{i+1}=v_{i \cdot} f$ with $f$ a constant factor.

Particle volume distribution is given by $q(z)$ and its integral represents the total volume of particle $Q$ per unit plasma volume.

$$
Q=\int q(z) d z
$$

The sectional model assumes a constant particle volume distribution $q(z)$ in a section $/$ :

$$
q(z)=\bar{q}_{l} \text { on }\left[\mathrm{z}_{1}-\mathrm{z}_{1+1}\right]
$$

The total number density on each section $/$ is thus given by :

$$
N_{l}=\int_{z_{l}}^{z_{l+1}} \frac{q(z)}{\exp (z)} d z \approx \int_{z_{l}}^{z_{l+1}} \frac{\bar{q}_{l}}{\exp (z)} d z=Q_{l} \frac{\exp \left(-z_{l}\right)-\exp \left(-z_{l+1}\right)}{z_{l+1}-z_{l}}
$$

In each section $I$, characterized by a size $I$ and an average charge $q_{l}$, we solve a continuity equation for the total particle volume $Q_{l}$ taking into account the section gain/loss rate due to coagulation, cluster sticking, transport and particle nucleation. 
This manuscript was accepted by J. Appl. Phys. Click here to see the version of record.

$$
\frac{\partial Q_{l}(t)}{\partial t}={\frac{\partial Q_{l}(t)}{\partial t}}_{\text {coag }}+{\frac{\partial Q_{l}(t)}{\partial t}}_{\text {sticking }}+S_{\text {nucleation }}-\nabla F_{l}
$$

The source term, $S_{\text {nucleation, }}$ corresponds to the nucleation rate and is obtained from the cluster module. It is non-zero for the only smallest size section.

The estimation of the coagulation source term, $\frac{\partial Q_{l}(t)}{\partial t}$ coag , in such a sectional model was extensively described in [20]. It involves several contributions:

- A particle gain through the coagulation between particles belonging to sections $i$ and $j$ with $i<l$ and $j<l$ that results in a particle that belongs to section $I$,

- A particle loss due the coagulation of particle belonging to section / with a particle of section $i$ with $i<1$,

- A particle loss due to the coagulation with two particles of section I,

- A particle loss due to the coagulation with particle of section $j$ with $j>l$.

This leads to the following master equation :

$$
\begin{aligned}
\left.\frac{\partial Q_{l}(t)}{\partial t}\right|_{\text {coag }} & =\frac{1}{2} \sum_{i=1}^{l-1} \sum_{j=1}^{l-1} Q_{i} Q_{j} w^{-1}(q(i), q(j)) \beta_{i, j, l}-\sum_{i=1}^{l-1} Q_{i} Q_{L} w^{-1}(q(i), q(l)) \beta_{i, l}^{2} \\
& -\frac{1}{2} Q_{L}{ }^{2} w^{-1}(q(l), q(l)) \beta_{l, l}^{3} \\
& -\sum_{j=l+1}^{n_{\max }} Q_{j} Q_{L} w^{-1}(z, q(l)) \beta_{i, l}^{4}
\end{aligned}
$$

where $\beta_{i, j, l}^{1}$ is the production coagulation coefficient between particles belonging to sections $I$ and $j$ that produce particle in section $I$ for neutral particles and $\beta_{m, n}^{x} x=[2,4]$ is the consumption coagulation coefficient between particles belonging to sections $m$ and $n$ [20]. $N_{\max }$ is the mumber of the largest section. The coagulation coefficients are obtained from ordinary neutral Brownian coagulation coefficient in free-molecule regime assuming spherical particles [5]. $w$ represents a charge correction factor that allows taking into account the impact of electrostatic interaction between the particles on the coagulation rate constant [5]. The coagulation is strongly enhanced for particles with opposite charges and strongly reduced for particles with the same charge. For example coagulation rate of $1 \mathrm{~nm}$ particles carrying only 1 elementary charge is reduced by more than 6 orders of magnitude as compared with coagulation rate of two neutral particles of the same size. On the opposite, coagulation rate of $1 \mathrm{~nm}$ particle carrying +1 and -1 elementary charge is increased by 2 orders of magnitude.

As mentioned previously, to take into account the charge fluctuation that takes place in electrical discharges, we assumed that the charge distribution in each section follows Matsoukas model [15]. More specifically, anticipating on the results section and for the discharge conditions considered here, only very small particles with size just above the nucleus diameter, i.e., with a diameter in the range 1-5 nm, may become neutral or 
positively charged [27]. Furthermore, the fraction of particles that may acquire more than one positive charge remains very weak over all the size distribution and can be disregarded.

Consequently, for each section I with an average negative charge $\bar{Z}_{l}$, the model developed here takes into account the particle fraction having a neutral $\left(\psi_{0,1}\right)$ or a single positive instantaneous charge $\left(\psi_{1,1}\right)$. Although neutral and positively charged particles represent only very small fraction of the particle cloud, coagulation is essentially driven by collision between these neutral and positively charged particles on one hand and the large majority of negatively charged particles on the other hand. Then, considering coagulation of particle of section $i$ with a particle of section $j$, collision can occur between [20]:

- the fraction $\psi_{0, i}$ of neutral particles of section $i$ with all particle of section $j$,

- the fraction $\psi_{l, i}$ of positive particle of section $i$ with neutral particle of section $j\left(\psi_{0_{j}}\right)$,

- the fraction $\psi_{l, i}$ of positive particle of section $i$ with negative particle of section $j\left(\psi_{1, j}\right)$ where coagulation rate is enhanced by attraction between two particles of opposite charge as described in [5].

The coagulation between two negatively charged particles is neglected due to the strong reduction of the coagulation rate by electrostatic repulsion, i.e., the electrostatic reduction factor $w$ is huge.

Following a similar approach, molecular sticking may affect the particle population of section I in three different ways:

- sticking takes place on a particle lying in section / with the resulting particle still belonging to section $/$ : this is called the intra-sectional sticking,

- molecular sticking takes place on a particle lying in section / with the resulting particle outside section $I$, which represents a loss for this section,

- molecular sticking takes place on a particle in section $1-1$ with the resulting particle still belonging to section $I$.

Consequently, the change of particle volume, $Q_{l}$, due to sticking can be written as :

$$
\left.\frac{\partial Q_{l}(t)}{\partial t}\right|_{\text {sticking }}=\underbrace{\bar{H}_{l} Q_{l}}_{\text {intra }}-\underbrace{\bar{I}_{l+1}}_{\text {sectional }}+\underbrace{\bar{I}_{l}}_{\text {gain }}
$$

The intra-sectional gain operator represents a first order moment of the sticking operator [21]. It is given by :

$$
\bar{H}_{l}=\frac{\int_{z_{l}}^{z_{l+1}} q(x) H(x) d x}{\int_{z_{l}}^{z_{l+1}} q(x) d x}=\frac{1}{z_{l+1}-z_{l}} \int_{z_{l}}^{z_{l+1}} H(x) d x
$$

The sticking operator $H$ for a single particle is defined by : 


$$
H=\gamma_{s t} \frac{1}{4} \sqrt{\frac{8 k T}{\pi m_{\text {cluster }}^{i}}} \frac{n_{i}^{z} m_{\text {cluster }}^{i}}{\rho_{p}} \cdot \frac{S_{p}}{v_{p}}
$$

with $\gamma_{s t}$ the molecular sticking coefficient, $n_{i}{ }_{i}$ density of $\mathrm{i}^{\text {th }}$ cluster of charge $z$ and with $m_{\text {clsuter }}^{i}$ mass. $\rho_{p}$ represents the carbon material density and $S_{p}$ is the surface of a single particle.

Eventually the inter-sectional operator is defined by:

$$
\bar{I}_{l+1}=\frac{\bar{H}_{l} Q_{l}}{1-\frac{\bar{m}_{l}}{\bar{m}_{l+1}}}
$$

Eq 9

where $\bar{m}_{l}$ is the averaged particle mass in section $l$.

The transport fluxes are estimated on the basis of a drift-diffusion model:

$$
\vec{F}_{l}=-D_{l} \vec{\nabla} Q_{l}+\bar{Z}_{l} \mu_{l} Q_{l} \vec{E}
$$

As mentioned previously, we made the assumption that particle charge distribution for each section follows a Gaussian distribution around an averaged charge as described by Matsoukas and al [15]. Using this assumption, we only solve for the averaged particle charge for each section following the same approach as the one described in [3]. More precisely, we solve for a charge balance equation taking into account particle charging by OML fluxes, transport, charge-change by coagulation and sticking of negative clusters. 


\section{Results}

\section{III.1. A brief description of the main plasma characteristics and cluster growth kinetics.}

Before discussing the detailed aerosol dynamics which is the main subject of this paper, we first give brief illustrations of the main peculiarity of the discharge characteristics and nucleation kinetics as inferred from previous model.

Typical plasma and potential distribution obtained in the investigated discharge are depicted in figure 2 that shows the electric field and the electron density as function of the distance from the cathode in the gap region. The electric field in the sheath is not shown in this figure. We distinguish the maximum of the electron density at the position of the electric field reversal, $2 \mathrm{~cm}$ from the cathode. The very slight field reversal, i.e., around $5 \times 10^{-2} \mathrm{Vcm}^{-1}$, in the NG turns out to be very efficient in providing an electrostatic potential structure where negative species can be trapped [3].

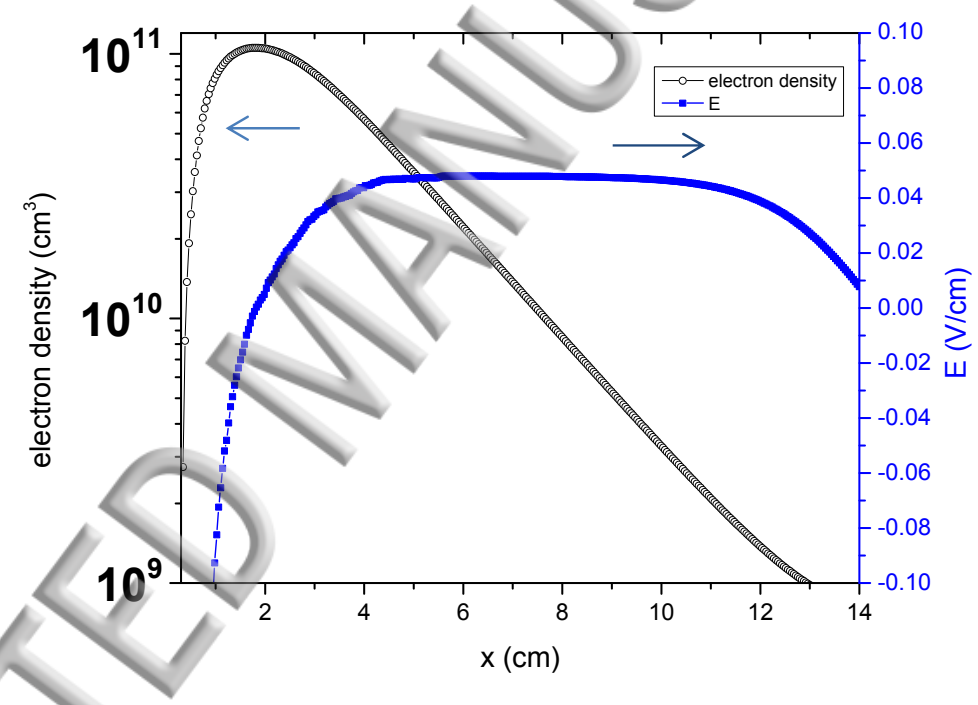

Figure 2 : Electron density, $\mathbf{n}_{\mathrm{e}}$, and electric field, $\mathbf{E}$, as functions of the distance to the cathode. The anode is located at $14 \mathrm{~cm}$ from the cathode.

Figure 3a shows the axial profiles of the density for the carbon atoms sputtered from the cathode and for different discharge durations [4]. C-atom shows a higher density-value at the cathode, i.e., $10^{11} \mathrm{~cm}^{-3}$, and the density decreases when clusters are transported toward the anode. Note that $C_{2}$ and $C_{3}$ clusters which are also produced by sputtering of the graphite cathode present similar profiles with density-values one order of magnitude smaller than $\mathrm{C}$ due to their reduced sputtering yields [4].

Figure 3a shows that the decrease of carbon cluster density with the distance to the cathode in the vicinity of the cathode, i.e., $x=0-2 \mathrm{~cm}$, is much pronounced for longer discharge 
durations. This is due to the increase of carbon particle density (see figure 7) and a subsequent enhancement of $\mathrm{C}$-atom loss through sticking at the particle surface near the field reversal position with discharge duration [3].

Figure $3 \mathrm{~b}$ shows the variation of $\mathrm{C}_{4}, \mathrm{C}_{4}{ }^{-}, \mathrm{C}_{15}$ and $\mathrm{C}_{15}{ }^{-}$densities with the distance to the electrode for discharge duration of $300 \mathrm{~s}$. For these clusters that result from molecular growth, i.e. not sputtered from the surface, negatively charged clusters density largely exceeds neutral cluster density. This shows the efficiency of the electrostatic trapping despite the very small value of the field reversal in the NG. In fact, we previously showed that clusters growth pathway starts with the sputtering of the neutral clusters $C_{1} C_{2}$ and $C_{3}$ followed by a limited aggregation of the small neutral clusters that leads to the formation of $\mathrm{C}_{4}-\mathrm{C}_{6}$ clusters that undergo attachment processes [3] . Once $\mathrm{C}_{4}^{-}$to $\mathrm{C}_{6}^{-}$negative clusters are produced, they are trapped in the field reversal region and grow up to the Largest Molecular Edifice that leads to particle nucleation [3].
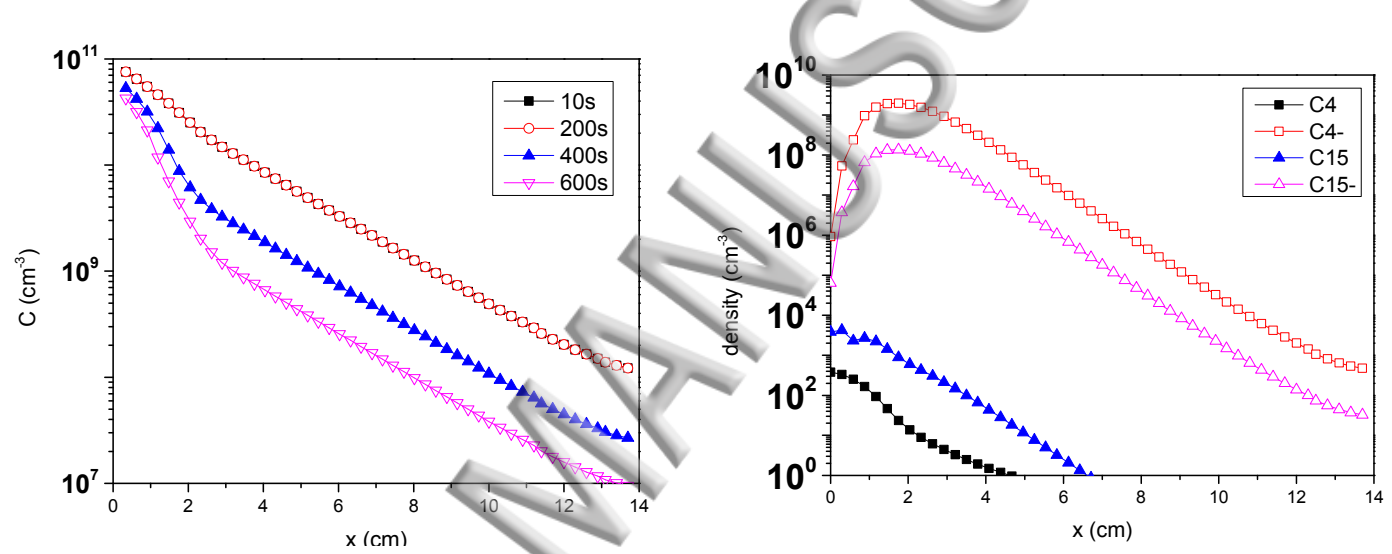

Figure 3 : (a) Carbon atom density as a function of the distance to the cathode for different discharge durations. (b) of $\mathrm{C}_{4}, \mathrm{C}_{4}, \mathrm{C}_{15}$ and $\mathrm{C}_{15}{ }^{-}$densities as functions of the distance to the cathode for $300 \mathrm{~s}$ discharge duration

Figure 4 shows the axial profiles of the nucleation rate along the discharge for different discharge durations. We observe that a time-period of $50 \mathrm{~s}$ is necessary to obtain a significant particle formation. This delay represents an incubation time for particle formation and corresponds to the characteristic time of the molecular growth of the first sputtered clusters up to the largest molecular edifice. Particle nucleation rate increases steadily between $50 \mathrm{~s}$ and $100 \mathrm{~s}$. It reaches a maximum value at $100 \mathrm{~s}$ and slightly decreases for longer discharge durations. This slight nucleation decrease is due to the fact that once particle are produced, a significant fraction of the sputtered $C, C_{2}$ and $C_{3}$ clusters are consumed by surface sticking on the particle, which limits molecular growth and nucleation kinetics. Nevertheless, due to the continuous graphite cathode sputtering and molecular growth, the nucleation rate remains significant for all discharge durations. 


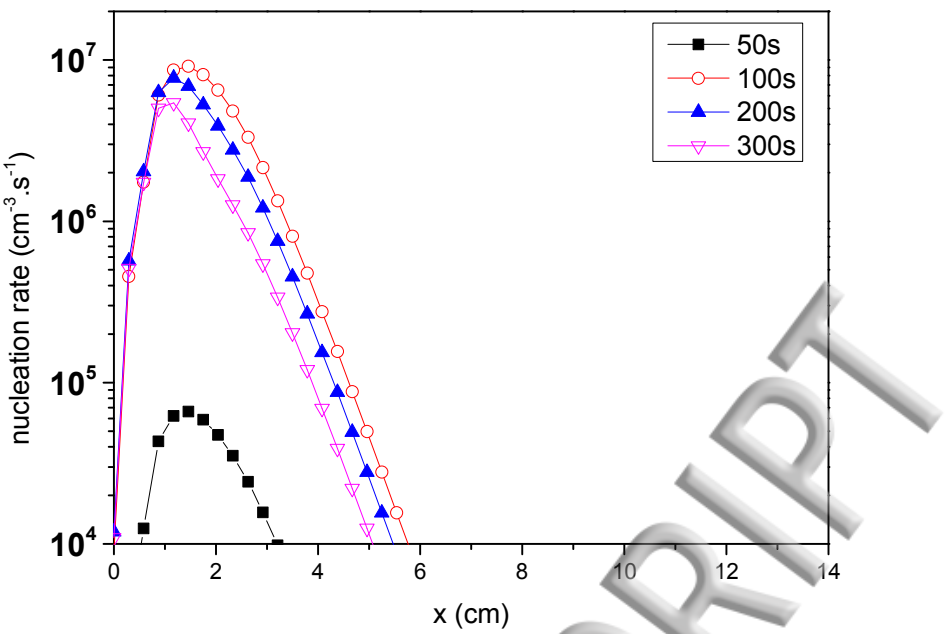

Figure 4 : Nucleation rate in $\mathrm{cm}^{-3} \mathrm{~s}^{-1}$ as a function of the distance to the cathode for different discharge duration values.

\section{III.2. Aerosol dynamics and particle size distribution in the investigated plasmas}

The aerosol dynamic equations were solved in the range 1-100 nm in order to achieve satisfactory estimates of the size distribution for the smallest sections, i.e., just above the nucleation threshold, that are especially involved in the coagulation process. The aerosol dynamic is governed by $2 \times 100$ master equations, 100 equations in the form of equation (4) for particle volume density and 100 governing equations for the average charge. The space derivative, i.e., the flux terms, were discretized over the electrode gap using a 100 points-1D space grid. The diffusion fluxes were approximated by a second order central difference, while the drift fluxes were approached by an upwind scheme. This results in a large nonlinear system of ordinary differential equations where the unknown are the particle volume density and average charge at a given grid point of the electrode gap. Starting with a very low density, almost zero, seed particle clouds, and using the nucleation rate and the plasma characteristics inferred from the cluster growth model and the plasma model discussed in the previous subsections, the governing equation were time-integrated using a fully implicit Backward Difference Formula (BDF) method during $300 \mathrm{~s}$.

The time-evolution of particle size distribution at each grid point in the electrode gap is described in the first part of this subsection. Then, we compare the results obtained from the aerosol models, i.e., size distributions, particle density, mean diameter, etc., with those determined experimentally or estimated from a single size aerosol model. In the last part of this section we analyze the relative predominance of the different phenomena, i.e., nucleation, surface sticking processes, coagulation and transport involved in the aerosol dynamics. 
This manuscript was accepted by J. Appl. Phys. Click here to see the version of record.

\section{III.2.a. Space-time evolution of particle size distribution.}

Figure 5 shows the particle size distribution, $\left(d N_{p} / d \log D_{p}\right)$ between the cathode $(\mathrm{x}=0)$, where clusters are sputtered, and the anode $(x=14 \mathrm{~cm})$ for discharge durations of $100 \mathrm{~s}, 150 \mathrm{~s}, 200 \mathrm{~s}$ and $300 \mathrm{~s}$. The particle density is reported in a logarithmic scale.

At $100 \mathrm{~s}$, we find particles everywhere outside the cathode sheath, i.e., above $0.3 \mathrm{~cm}$ from the cathode. The major fraction of the particle cloud is however located around the field reversal position, i.e., $\mathrm{x}=1.8 \mathrm{~cm}$. This is indeed the position where the particle population is maximum for all the size values. We also observe that the particle cloud is much more localized for larger particle diameter, which means that the electrostatic trapping effect is much more pronounced for larger particles. As a matter of fact, the population of few $\mathrm{nm}$ size particles tends to be significant over all the discharge gap, larger particle population shows significant population in a limited region around the field reversal position. For example, the $10 \mathrm{~nm}$ diameter particles cloud extends from the sheath edge to a distance of $6 \mathrm{~cm}$ from the cathode, while the $20 \mathrm{~nm}$ diameter particle cloud is more localized in the range $x=1-3 \mathrm{~cm}$. Figure 5 also shows that after $100 \mathrm{~s}$ discharge, the maximum density is obtained for a particle diameter of $10 \mathrm{~nm}$, a value that represents the mean diameter for this discharge duration. Despite the pronounced maximum population around $10 \mathrm{~nm}$ diameter, it appears that all the size distribution, especially the large size region of the distribution, is populated in the vicinity of the field reversal position. In fact, the large particle observed at this position corresponds to the initial seed particles that were trapped in the field reversal region where they could grow and reach diameter as high as $100 \mathrm{~nm}$.

The structure of the particle cloud dramatically changes for a discharge duration of $200 \mathrm{~s}$ and above. Indeed for this discharge duration the particle cloud splits in two populations (modes). We first have a few nm size, i.e., 1-3 nm, particle cloud that spread over almost all the discharge gap, see the very thin colored line in the vicinity of $d=0 \mathrm{~nm}$ with a $d N_{p} / d L n\left(d_{p}\right)$ values that range between $10^{4}$ and $10^{6}$ and that extends over the whole discharge gap. Then we have a second mode that contains the major fraction of the particles and that is strongly confined around the field reversal position. In this mode, the particles show size-values ranging between 15 and $100 \mathrm{~nm}$. The size distribution reaches a maximum value slightly greater than $10^{8} \mathrm{~cm}^{-3}$, just at the position of the field reversal and for a size value of 35$40 \mathrm{~nm}$. A major characteristics of the size distributions obtained for discharge durations above $200 \mathrm{~s}$, is the very strong population depletion that starts just above a size value of 2$3 \mathrm{~nm}$ and may extend up to a size of 15-20 nm depending on the discharge duration.

To appreciate this depletion in the size domain ranging between 3 and $20 \mathrm{~nm}$, we report in figure $6 a$ the size distribution obtained for discharge durations of 100s, 200s, and $300 \mathrm{~s}$ at the field reversal position, i.e., $x=1.8 \mathrm{~cm}$. Figure $6 \mathrm{~b}$ shows the details of the size distribution in the depletion region, i.e., for $d_{p}=1-10 \mathrm{~nm}$. Figure 6 a confirms that for a short discharge duration, i.e., below $100 \mathrm{~s}$, the size distribution increases steadily up to a maximum value, obtained for a particle diameter of $10 \mathrm{~nm}$, and then decreases for larger diameter values. It 
is worthy to mention here that the population of the smallest size section, i.e., just above the nucleation threshold, is quite significant and only one order of magnitude smaller than the maximum size distribution. Figure 6a shows the very sharp decrease of the size distribution just above the nucleation threshold for long discharge durations. For instance, for a discharge duration of $200 \mathrm{~s}$, the particle size distribution decreases by more than three orders of magnitude when the particle size increases from $1 \mathrm{~nm}$ (the nucleation threshold) to $1.5 \mathrm{~nm}$. A sharpest decrease is even observed for longer discharge durations. As a matter of fact, for $300 \mathrm{~s}$ discharge duration, the size distribution decreases by more than 6 orders of magnitude over 1-1.5 $\mathrm{nm}$ size range. Further, particle depletion tends to take place over much larger size range for longer discharge durations. As a matter of fact, the size range for which the size distribution is two orders of magnitude smaller than its maximum value increases from 2-10 $\mathrm{nm}$ for a discharge duration of $200 \mathrm{~s}$ to $2-20 \mathrm{~nm}$ for a discharge duration of $300 \mathrm{~s}$. This enlargement of the size range over which the depletion of the particle population takes place is obtained along with the increase of the size at which the distribution reaches its maximum. This increases from $30 \mathrm{~nm}$ to $40 \mathrm{~nm}$ when the discharge duration increases form $200 \mathrm{~s}$ to $300 \mathrm{~s}$.

The distributions obtained in this work show similar qualitative behavior as those obtained by Kim and Kim [14] in CCRF dusty silane plasmas. As a matter of fact, although the discharge conditions are quite different, a strong depletion build-up, similar to the one obtained here, for the intermediate sizes was also reported by these authors who used a one-dimensional aerosol model with prescribed electron density and temperature [14]. Particle distributions with a strong depletion were also obtained in the gap center of CCRF dusty silane plasmas by Agarwal and Girshick who used a fully selfconsistent model for the plasma and the aerosol dynamics [13]. Note that in these two references the size distribution maxima at the nucleation threshold and for the larger population, i.e., 10-50 nm diameter population, show comparable values. This represents a significant difference with respect to the results obtained in our conditions for which the population at the nucleation threshold is several orders of magnitude smaller than the large particle population. This difference may be due to the fact that the authors of these two references made use of constant prescribed nucleation rates that are probably much greater than the nucleation rate calculated from the molecular growth model used in this work. It is worthy to mention that depletion-free monotonous distributions was obtained by De Bleeker et al. who used a one-dimensional self-consistent model of plasma and aerosol dynamics in CCRF silane plasmas [28]. We believe that this difference is due to the fact that in the discharge conditions investigated by De Bleeker et al., the population of large particles remains very limited and coagulation takes place only between small size particles.

To conclude this first part, we can say that the detailed aerosol dynamic simulations show that for long discharge durations when the particle cloud starts to be highly populated and particle mean diameter reaches significant values, the particle size distribution shows two populations: a very small size, i.e., just above the nucleation threshold, population and a 
major large size population that defines the average characteristics of the particle cloud. The size distribution is characterized by a strong depletion in a size domain starting just above the nucleation threshold and that may extend up to $20 \mathrm{~nm}$ diameter.
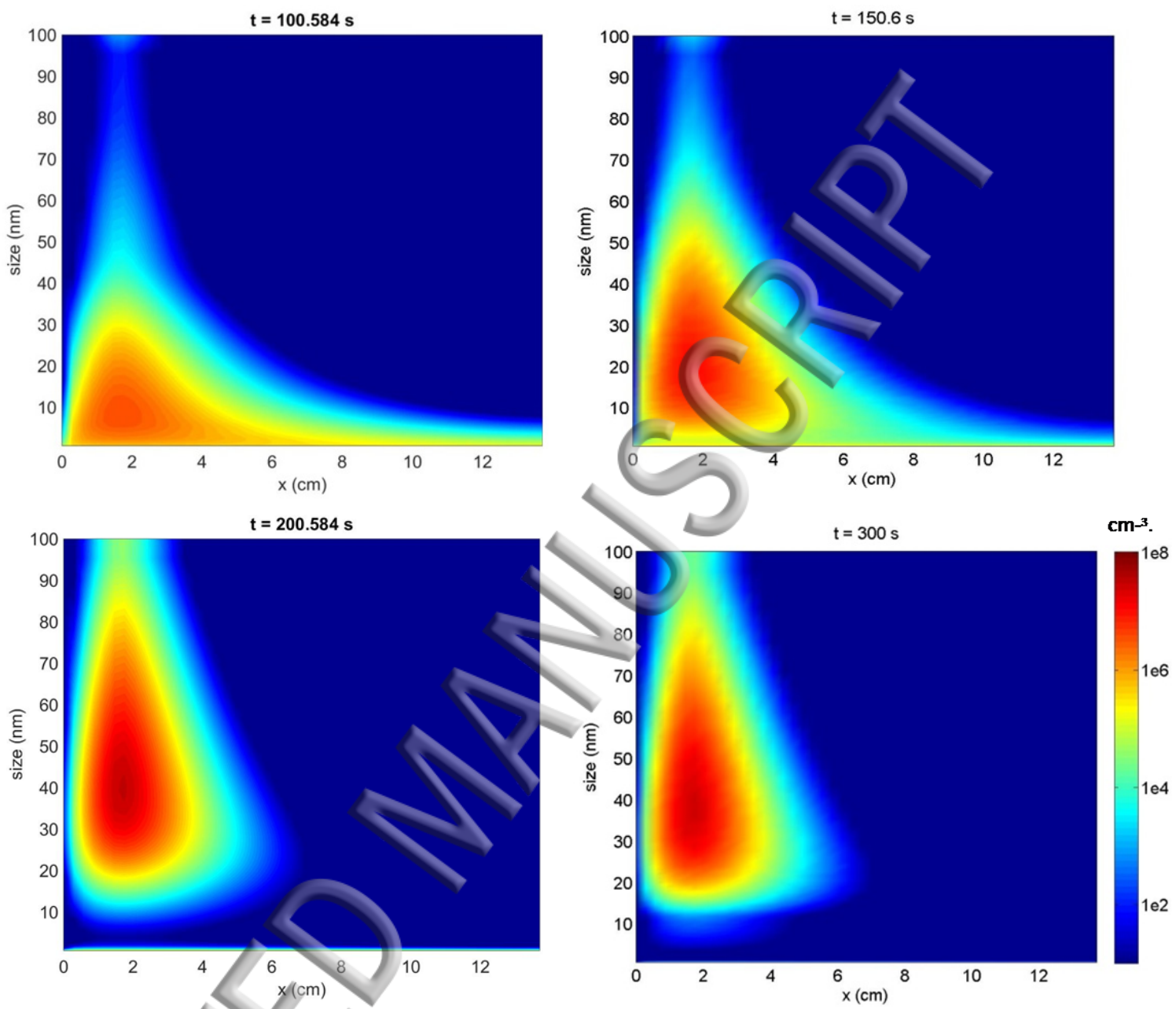

Figure 5 : Particle size distribution $\left(d N_{p} / d \log (D p)\right)$ in the electrode gap region at $100 \mathrm{~s}(\mathrm{a}), 150 \mathrm{~s}$, $200 \mathrm{~s}$ and $300 \mathrm{~s}$. The colors gives the magnitude of the size distribution in a logarithmic scale

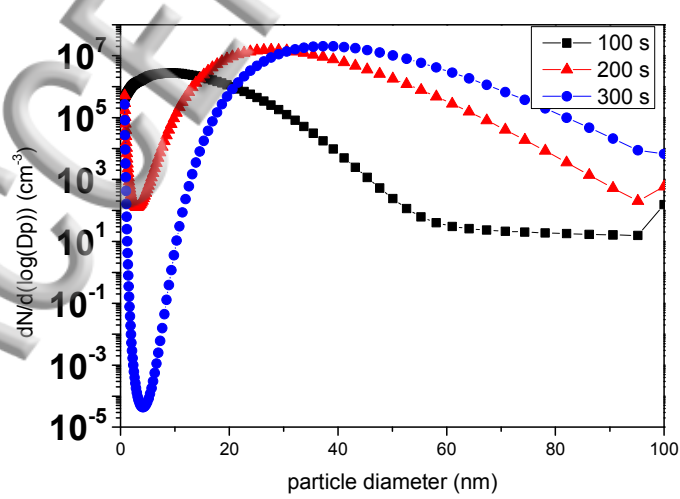

(a)

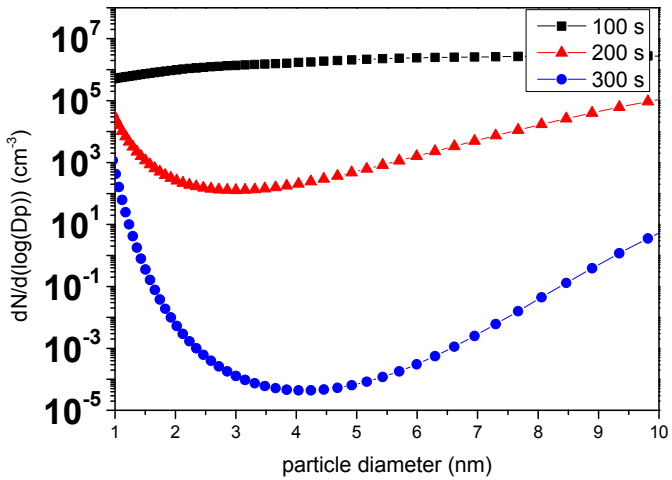

(b) 
This manuscript was accepted by J. Appl. Phys. Click here to see the version of record.

Figure 6 : Particle size distribution at the field reversal position, i.e., at $1.8 \mathrm{~cm}$ from the cathode, for discharge durations $(b)$ is a zoom on the range 1-10 $\mathrm{nm}$.

\section{III.2.b. Model validation}

The calculated size distributions were used to determine the number density and the meandiameter of the particle cloud. Figure 7 shows the time-variations of the particle number density at the field reversal position calculated by the sectional model and the single size model. The particles reach a significant density after discharge duration of $50 \mathrm{~s}$, which is consistent with a short characteristic time for the nucleation process as discussed in the previous section. The sectional model predicts a particle density-value smaller than that obtained from the single-size model over all the time range considered in the simulation. However, for discharge durations smaller than $100 \mathrm{~s}$, particle densities obtained by both models are quite similar. For longer discharge durations, the particle density determined using the sectional model reaches a plateau with a value of $10^{8} \mathrm{~cm}^{-3}$, which is one order of magnitude smaller than the density obtained, for the same conditions, by the single size model. Figure 8 shows the time-variations of the particle mean diameter calculated by both models. For the sectional model this value is obtained by a weighted averaged of the particle section with their corresponding density. The mean-diameter value predicted by the singlesize model remains always slightly larger than that predicted by the detailed aerosol model over all the investigated time range. However, the values obtained by the sectional model and the single size model shows only a slight difference for intermediate discharge durations, i.e., 100-200 s, and tends to get closer for longer discharge duration. As a matter of fact, the particle diameter predicted by the sectional model reaches a value of $30 \mathrm{~nm}$ after $200 \mathrm{~s}$ and $40 \mathrm{~nm}$ after $300 \mathrm{~s}$. For the same time period the mean diameter values predicted by the single size model are $35 \mathrm{~nm}$ and $42 \mathrm{~nm}$.

It is worthy to remind here that the particle nucleation rates and the cluster densities available for particle growth by sticking are exactly the same in the two models.

The particle density value of $10^{8} \mathrm{~cm}^{-3}$ predicted by the aerosol dynamic model for $300 \mathrm{~s}$ discharge duration is in a very good agreement with experimental measurements made by laser extinction [29] that yield a particle density value of approximately $2 \times 10^{8} \mathrm{~cm}^{-3}$ for a discharge duration of $600 \mathrm{~s}$. On the other hand, these density values are one order of magnitude smaller than the one obtained using the single size model. It appears therefore that considering a detailed aerosol dynamic model results in a substantial improvement in the prediction of the particle density. These comparisons also show how crucial is the use of a coagulation model that considers the detailed particle size-distribution along with charge fluctuation effect, in order to achieve a satisfactory prediction of the dust cloud density. Particle average diameter determined through experimental measurements by Scanning electron Microscopy carried out on the investigated device are also reported in Figure 8 . The particle collection procedure consists in using a high flow-rate pumping at the end of every discharge, in the direction of the gravity field. The aim is to collect the largest fraction of particles contained in the vessel on substrates. These substrates are located at the bottom 
of the vessel and are pushed one after the other, just below an aperture made in the anode. Each substrate is used for a given plasma duration. A set of several substrates provides the growth kinetics. Size distributions of Figure 9 and 10 were established with a bin width of 5 $\mathrm{nm}$, used for around 800 size measurements (in each case). The experimental points in figure 8 present the mean values of size distributions well fitted by log-normal distributions with 1.2-1.3 multiplicative standard deviations. A discussion was included in the revised version to clarify the measurement method.

These measurements showed that the particle mean-diameter reaches a value of approximately $35 \mathrm{~nm}$ after only $50 \mathrm{~s}$ discharge duration, $42 \mathrm{~nm}$ after $120 \mathrm{~s}$ and $45 \mathrm{~nm}$ after $300 \mathrm{~s}$. When comparing these values with those calculated using either the detailed aerosol dynamic model or the single size model one should remember that the absolute value of the calculated diameters are obtained assuming a particle mass density of $1 \mathrm{~g} . \mathrm{cm}^{-3}$ [5]. The actual particle mass density is however not really known and cannot be easily estimated. Therefore, quantitative comparison between calculated and measured diameters cannot be completely meaningful, and only qualitative comparison can be carried out. From this point of view, it appears that the particle diameter determined experimentally is significantly larger than those estimated by both the aerosol and the single-size models at the early stage of the particle formation just after the incubation time during which the nucleation takes place. Also, the particle growth kinetics predicted by the models appears to be significantly slower than that determined experimentally. Indeed, measurements shows that steady state value for the average diameter is reached after only $150 \mathrm{~s}$, while the steady state averagediameter value is not reached in the models even for $300 \mathrm{~s}$ discharge duration. Note However that the calculated time-variation of the average diameter tends to decrease for discharge durations above $250 \mathrm{~s}$ and calculated average diameter seems to approach the steady state value at this duration. In particular, the difference between the measured and calculated diameters tends to decrease for long discharge durations. The measured and calculated diameter values for $300 \mathrm{~s}$ discharge duration are in fairly good agreement. The difference between the models prediction and the experimental measurement seems to originate at least partly from the nucleation kinetics (molecular growth model) that strongly depends on the sputtering yield. Therefore this difference may be at least partly due to the uncertainty of the sputtering yield and the rate constants involved in the cluster growth model [4]. Nevertheless, both the detailed aerosol dynamic model and the single size model yield mean diameter values that are not far from those measured experimentally. 


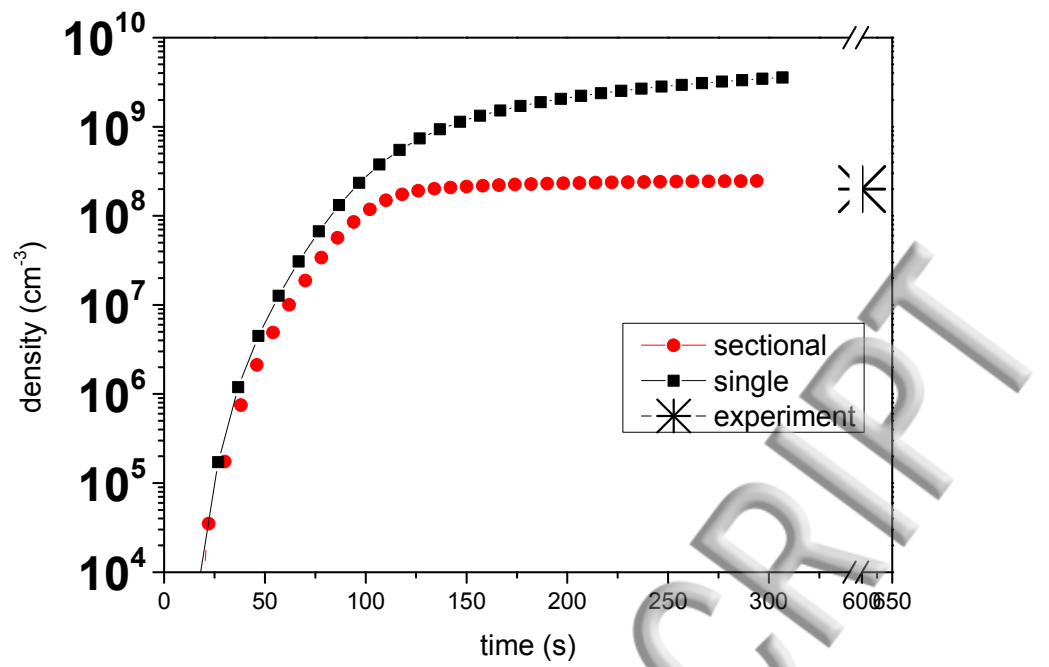

Figure 7 : Time evolutions of the particle density predicted using sectional model (circle) and a single-size model (square) at the field reversal . The particle density estimated experimentally at $600 \mathrm{~s}$ is also reported in the figure

Figure 8 : time evolution of the particle mean diameter with sectional model and averaged value model. The particle mean diameter estimated experimentally is also reported in this figure

To further validate the model prediction, at least qualitatively, we compare in this paragraph the measured and calculated particle size distributions. However, since, as mentioned previously, the estimation of the particle size is based on a prescribed qualitative value of the particle mass density, a direct quantitative comparison of the measured and calculated particle size distributions is not totally meaning full. It is indeed much more relevant to compare particle size distributions that correspond to the same mean particle diameter. Of course such comparison cannot validate the capability of the model to predict the absolute particle size distribution. It allows however obtaining at least a qualitative validation of the very peculiar particle size distributions that were calculated for the investigated discharge 
conditions. In other words, this validation concerns more specifically the shape of the calculated particle distribution.

Figure 9 and 10 show the calculated and measured particle size distributions corresponding to similar mean particle diameter of about $20 \mathrm{~nm}$ (figures 9-a and 9-b) and $40 \mathrm{~nm}$ (figures 10-a and 10-b). Comparison of the measured and calculated distributions shows that although the dynamic of particle growth predicted by the model shows a delay of approximately $100 \mathrm{~s}$ with respect to the experiment, i.e., the model predict that a mean diameter of $40 \mathrm{~nm}$ is reached at $275 \mathrm{~s}$ while this mean-diameter value is observed experimentally for a time period of $150 \mathrm{~s}$, the shape of the calculated and measured size distributions obtained for the same mean diameter are very similar.

For instance, Figures 9a and 9b show that for a short discharge-duration, both measured and calculated particle distributions are lognormal and centered on a single maximum corresponding to a size of $20 \mathrm{~nm}$. Figures $10 \mathrm{a}$ and $10 \mathrm{~b}$ are obtained for longer discharge durations. In this case, both experimental and calculated distributions show a log-normal shape with a population depletion for particle below $10 \mathrm{~nm}$, a sharp population increase in the size range between 15 and $40 \mathrm{~nm}$ and a much slower decrease for larger sizes. As discussed in the beginning of this section, see also figure $6 a$, beside the main population that corresponds to particles with size in the vicinity of the mean diameter, there is a second population with much smaller size and a fairly significant density that shows a populati*on maximum at the nucleation threshold. Although, this population could not be directly identified experimentally, its existence, which is necessary for enhancing the coagulation processes, is consistent with the particle density and structure observed experimentally. And indeed, experimental characterization by SEM showed that carbon particles obtained in the investigated discharge may contain 2-3 $\mathrm{nm}$ carbon particle agglomerates that are imbedded in a continuous carbon matrix [30],[31]. This finding is consistent with the growth mechanism as may be inferred from the present model with simultaneous coagulation process involving the small size distribution and an enhanced surface sticking process on the large particles (see the discussion in the next subsection).

To conclude this part, we can say that the particle size distributions predicted using the detailed aerosol dynamic model are in good qualitative agreement with those measured in the experiment. Further, the bimodal nature of the predicted distributions, with a very small size population, just above the coagulation threshold, and a major population with a size around the mean particle diameter, is consistent with the particle morphology and structure determined experimentally. 
(a)

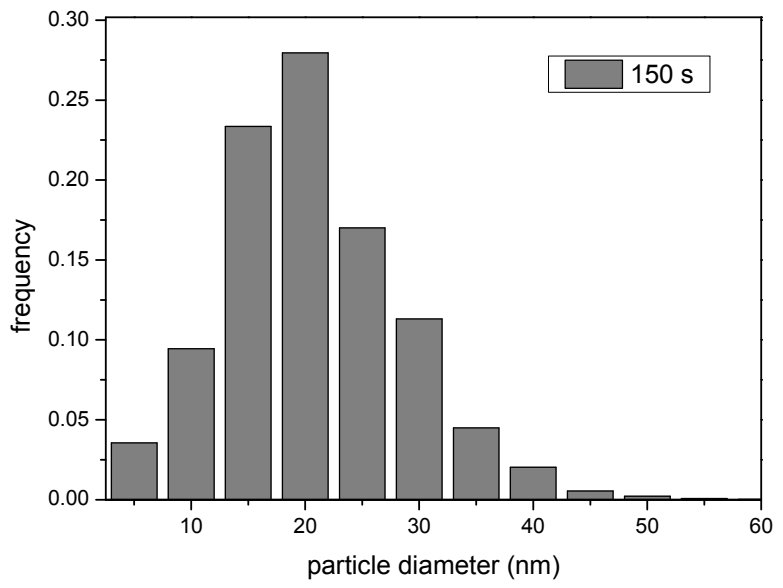

(b)

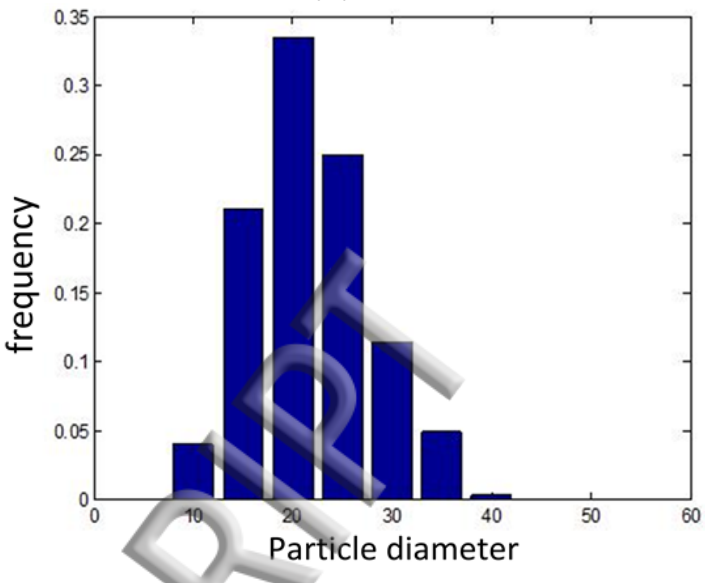

Figure 9 : Particle size distributions corresponding to a $20 \mathrm{~nm}$ mean diameter obtained from measurement (a) and calculated by the sectional model at the field reversal position, i.e., $x=1.8 \mathrm{~cm}$ (b). The measured distribution corresponds to a discharge duration of $45 \mathrm{~s}$ while the simulated distribution corresponds to a discharge duration of $150 \mathrm{~s}$.

(a)

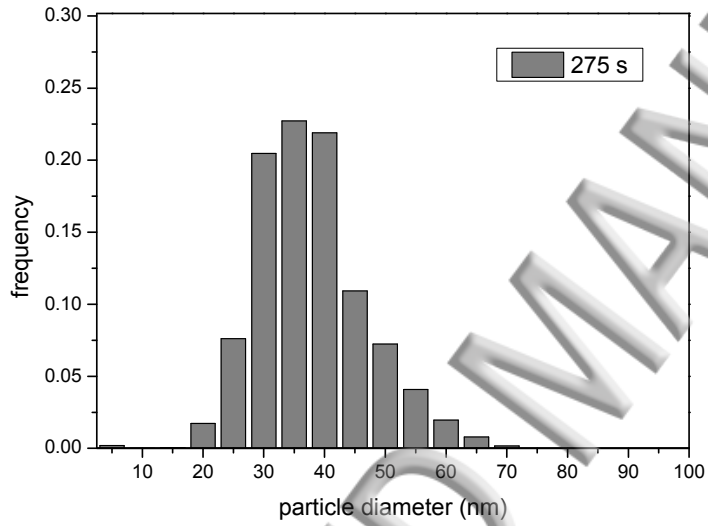

(b)

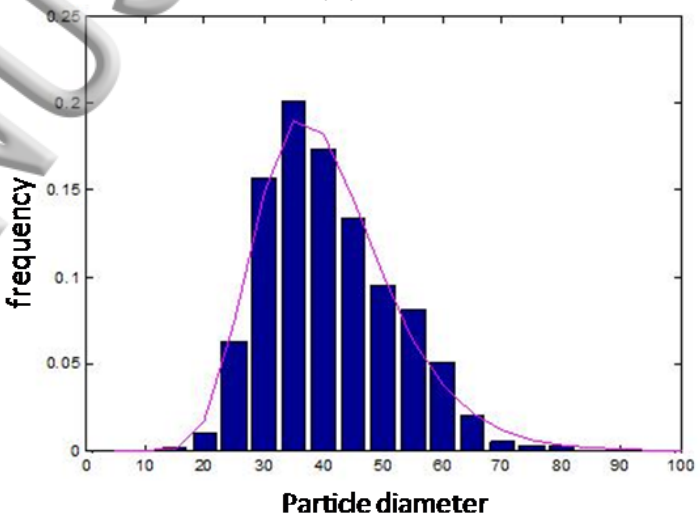

Figure 10: Particle size distributions corresponding to a $40 \mathrm{~nm}$ mean diameter obtained from measurement (a) and calculated by the sectional model at the field reversal position, i.e., $x=1.8 \mathrm{~cm} \mathrm{(b)}$. The measured distribution corresponds to a discharge duration of $120 \mathrm{~s}$ while the simulated distribution corresponds to a discharge duration of $275 \mathrm{~s}$.

\section{IIn2.c. Analysis of the different phenomena involved in the}

\section{aerosol dynamic}

The smaller particle density value obtained when using a detailed aerosol model (as compared with the single size model), may be easily explained by the capability of the aerosol dynamic model to accurately describe the enhanced particle coagulation kinetics between particles of different size and/or different charges. Figure 11a shows the particle size distributions obtained using the aerosol dynamic model with and without taking into account the coagulation process. Disregarding the coagulation processes, results in a truncated regular lognormal-like distribution with a single maximum around the mean diameter. The population depletion therefore disappears when coagulation is disregarded. 
This depletion effect is in fact very characteristic of the coagulation process that prevents the growth of small size particles that are rapidly consumed by coagulation with the particles belonging to the core of the distribution. As a result, intermediate size distribution cannot be produced and the distribution is depleted at intermediate sizes. In the absence of the coagulation processes, the particle population density is strongly enhanced. This may be easily seen in figure 11a that shows that the size distributions obtained when disregarding the coagulation processes is one order of magnitude larger than the one obtained when taking onto account this effect. Further, figure $11 \mathrm{~b}$ shows the time evolutions of the particle densities at the field reversal position calculated using the aerosol dynamic model with and without taking into account the coagulation processes. In the same figure we also report the time variation of the particle density calculated using the single size distribution model at the same position. We can clearly see that disregarding the coagulation process results in a larger particle density with values that are similar with those obtained using the single size distribution model.
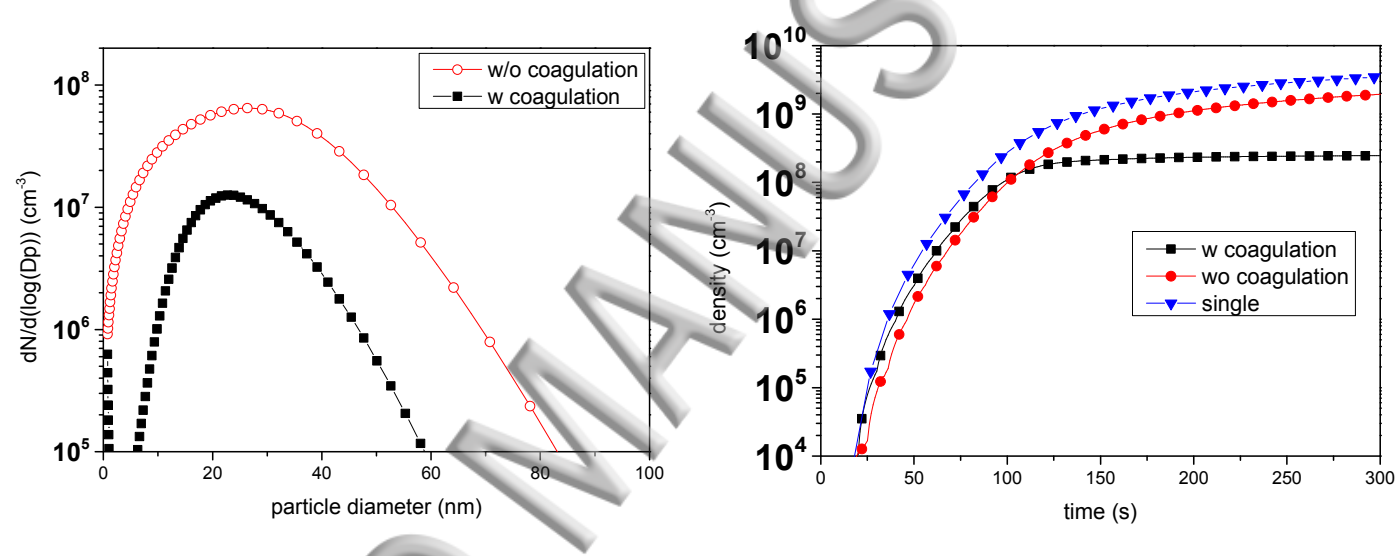

Figure 11 : (a) particle size distributions presenting similar averaged diameter obtained (1) with coagulation at $170 \mathrm{~s}$ (filled square) and without coagulation at $300 \mathrm{~s}$ (empty circle). (b) time-evolution of the particle density at the field reversal position calculated with and without taking into account the coagulation process. The density calculated with the single size model is also show.

To further understand how coagulation processes plays in affecting the size distribution, we report in figure $12 \mathrm{a}$ the particle volume gain per unit plasma volume due to coagulation, i.e., the positive part of the coagulation source term, as a function of the size. Surprisingly, the coagulation process seems to be very active during the early stage of the discharge, i.e., during the first $100 \mathrm{~s}$, when the mean diameter is still small and the population of the 1-5 nm size particles significant. For larger discharge duration when the particle size increases and the size distribution is depleted at the intermediate size, the coagulation rate experiences a dramatic decrease both for the very small and the core distribution particles. For instance the coagulation rate for the size-range $25-30 \mathrm{~nm}$ decreases by more than four orders of magnitude when the time increases from 200 to $300 \mathrm{~s}$. This decrease is well correlated to the strong depletion of the particle population in the range 1-5 $\mathrm{nm}$. In particular, particles with size ranging between 3 and $5 \mathrm{~nm}$ experience a population decrease by more than 6 
orders of magnitude (cf. figure 12a). In order to understand how the coagulation contributes to the mass balance of the different section, we show in figure $12 \mathrm{~b}$ the positive and negative parts of the coagulation source term for the different sections at the field reversal position and for a time period of $100 \mathrm{~s}$. Figure $12 \mathrm{~b}$ clearly shows that the net contribution of coagulation to the volume change for small particle is negative ( the loss is much greater than the gain), while this net contribution is positive for particles that show size-values above the mean-diameter. This result shows that coagulation tends to consume small particles and enhance the population of large particles, which-is consistent with the population depletion observed at the small size. The effect of coagulation on particles as function of their size may be well appreciated by looking to the frequency loss defined as the ratio of the negative part of the source terms of the aerosol dynamic equation and the size distribution, i.e., $\frac{\left(\frac{\partial Q}{\partial t}\right)^{-}}{Q}$. This is depicted in figure 13 that shows that the loss frequency of small particles is much larger than that of large particles. Further, the loss frequency of small particles tends to increase with the time while the opposite is observed for large particles. For instance, the loss frequency of small particles is greater by more than 6 orders of magnitude than the loss frequency of large particles for long time periods, i.e., 200 and $300 \mathrm{~s}$. This indicates that the coagulation processes induces a very large relative change of the small particle volume, while its effect on the volume of the large particles remains quite limited. In other words, the coagulation process involves a large number of small particles that interact with large particles. The change of large particles volume due to coagulation remains however quite limited.
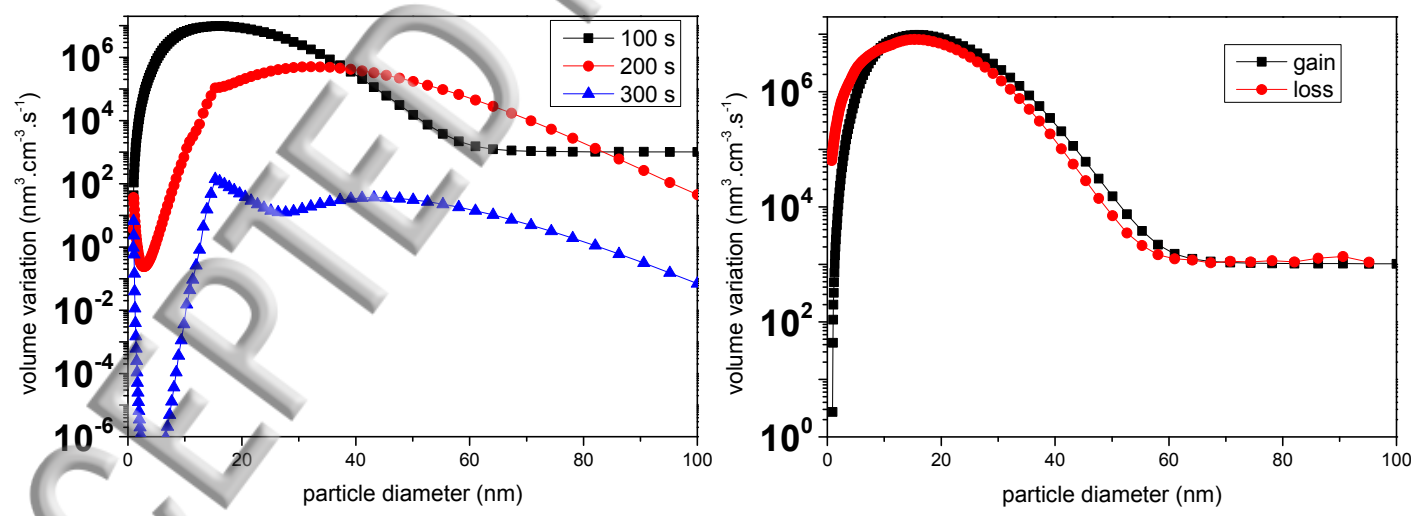

Figure 12: (a) particle volume gain per unit plasma volume as a function of the particle size for different time-period at the field reversal position (b) particle volume gain and loss per unit plasma volume as a function of the particle size for a time-period of $100 \mathrm{~s}$ at the field reversal position

In fact, the increase of the large particle diameter population and the subsequent shift of the size distribution toward the large size, is mainly insured by surface sticking processes. Figure 14 shows the particle volume gain per unit plasma volume through surface sticking 
processes as a function of the particle size. The contribution of surface sticking process to the particle growth kinetics exceeds the contribution of coagulation by more than one order of magnitude at $100 \mathrm{~s}$, four orders of magnitude at $200 \mathrm{~s}$ and eight orders of magnitude at $300 \mathrm{~s}$. This basically shows that for long discharge durations coagulation does not affect the core of the size distribution that is governed by surface sticking process. Coagulation essentially govern the populations of the very small size particles, i.e., near the nucleation threshold. Basically the coagulation process represents the major loss mechanism of small particles. This process balances particle production by nucleation and limits the increase of particle density. Therefore the coagulation process determines, along with the nucleation process, the overall particle density at steady state. It is worthy to mention here that when this process is not properly taken into account and underestimated, as in the single size model, steady state density can be hardly achieved. As a matter of fact, figure 7 shows that particle density predicted by the single size model keeps increasing over all the investigated range of discharge durations, while particle density predicted by the aerosol dynamic model rapidly reaches a steady state value.

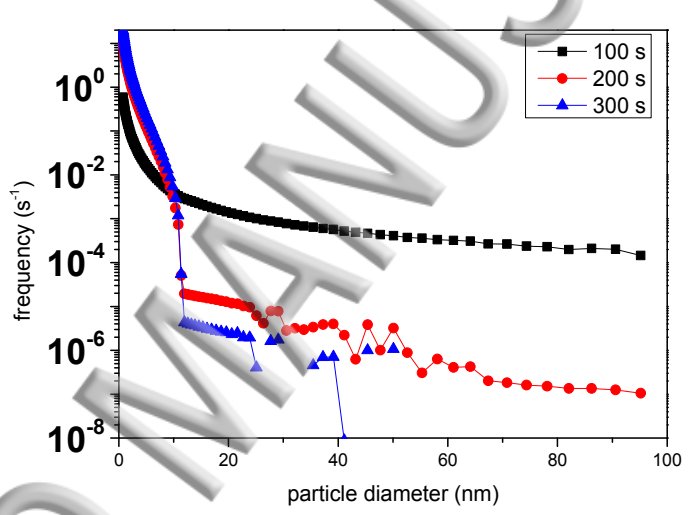

Figure 13: loss frequency of particles as function of the size for different time periods.

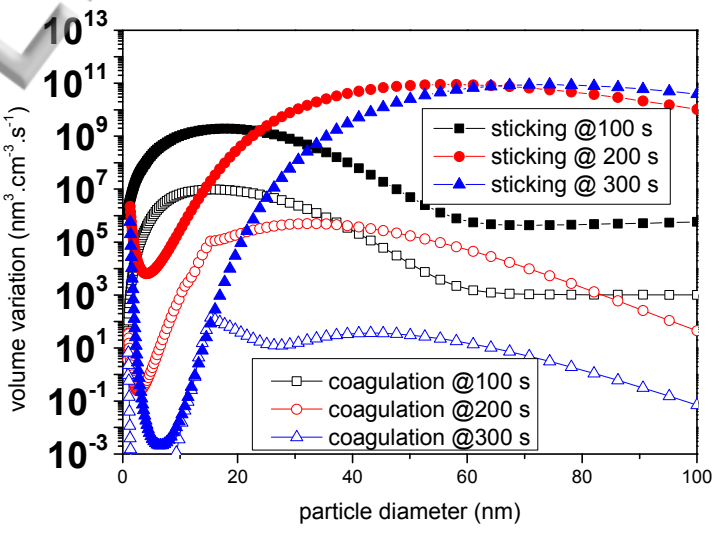

Figure 14 : Rate of particle volume gain through surface sticking processes as function of the particle size for different durations at the field reversal position 
As clearly shown in figure 1 , the coagulation between particles of very different size is strongly enhanced, i.e., coagulation rate between particle with a size ratio of $10^{2}$ is three orders of magnitude larger than coagulation rate between particles with the same size. Beside this size effect, charge fluctuation may result in a substantial fraction of neutral and positively charged particles, especially when the average charge is limited. As a result, the coagulation can be also enhanced by electrostatic attraction between particles with opposite charges. Therefore, the main drivers for coagulation process are both the size effect and the charge fluctuation effect. This later essentially involves small-particles and may be appreciated in figure 15 where we showed the evolution of the average charge (Figure 15 a) and the fraction of neutral and positively charged particles (Figure 15b) as a function of the particle size at the field reversal position. Charge fluctuation involves particles with diameter smaller than $10 \mathrm{~nm}$ and average charge below 2 elementary charges. This means that particles with diameter as high as $10 \mathrm{~nm}$ will contribute to coagulation with particles of the core distribution. Note however that neutral and positively charged particles represent more than $5 \%$ of the total particles only for size values below $5 \mathrm{~nm}$. Therefore particles with sizevalues between the nucleation threshold and $5 \mathrm{~nm}$-diameter bring the main contribution in the coagulation and represents therefore the driver of this process.

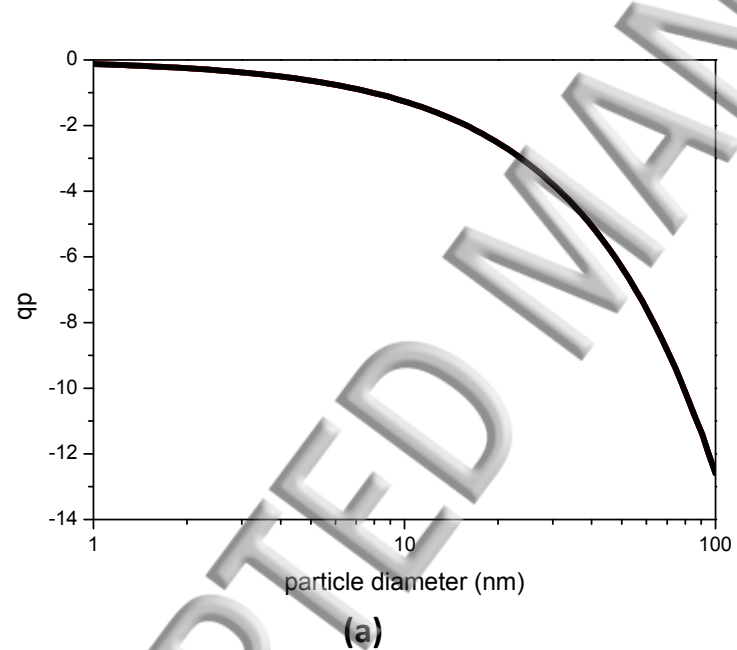

Figure 15 :

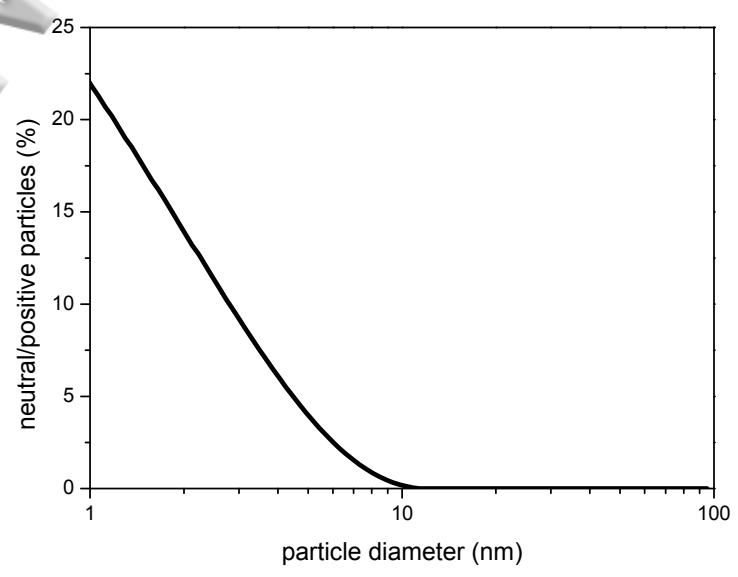

(b)

Averaged charge (a) and percentage of neutral and positively charged particles (b) as functions of the particle size

The average charge carried by the major part of the particle population, i. e., which shows a diameter value around $40 \mathrm{~nm}$, is approximately 5 elementary charges. This average charge value gives, for a particle density of $10^{8} \mathrm{~cm}^{-3}$, cf. figure $11 \mathrm{~b}$, a global charge of $5 \times 10^{8}$ elementary charges per $\mathrm{cm}^{3}$. This is much lower than the electron density which is in the range $10^{10}-10^{11} \mathrm{~cm}^{-3}$ in the negative glow of the investigated discharges (Figure 2 and reference [3]). This supports the assumption made in this article with respect to the negligible contribution of the solid particles to the overall plasma equilibrium. The average charges calculated in this work compare well with those reported by Kim and Kim for CCRF dusty silane plasma. They found a value of approximately 5 elementary charges for $40 \mathrm{~nm}$ - 
diameter particles assuming an electron temperature of $2 \mathrm{eV}$. It also compares well with the average charge values reported by Agarwal et al. who used a self-consistent onedimensional model with prescribed nucleation rate to describe similar (CCRF) silane discharges. De Bleecker et al. who used a self-consistent one-dimensional model for (CCRF) dusty silane discharges, obtained similar average charge values for particles located in the center of the discharge gap where the electron temperature is usually moderate $(2 \mathrm{eV})$ and a much larger charge values, i.e., 100 elementary charges, in the vicinity of the sheath where the electron temperature is usually very large.

Transport effects also contribute significantly to the space-time evolution of the particle cloud that extends from $x=1 \mathrm{~cm}$ to $x=3 \mathrm{~cm}$ distance from the cathode. The nucleation, coagulation and surface sticking rates are not uniform in the discharge gap. The analysis of the net transport fluxes and the coagulation and surface sticking source terms show that many of the small particles that contribute to the coagulation process in the bulk of the dust cloud, i.e., in the vicinity of the field reversal region located at $x \approx 1.8 \mathrm{~cm}$, come from the periphery of the dust cloud, i.e., $x \approx 1-1.5 \mathrm{~cm}$ and $x \approx 2-2.5 \mathrm{~cm}$. This may be well understood by looking at Figure 16a that shows the rate of change due to transport fluxes in the axial direction of the small particles with size below $5 \mathrm{~nm}$. It appears that small particles are transported by diffusion from the cloud periphery, i.e., $x \approx 1-1.5 \mathrm{~cm}$ and $x \approx 2-2.5 \mathrm{~cm}$, to the cloud center, i.e., $x \approx 1.8 \mathrm{~cm}$. In fact, particle nuclei are produced and undergo a first growth through surface sticking of $C, C_{2}$ and $C_{3}$ neutral species sputtered from the cathode at the periphery of the particle cloud where particle density and coagulation rate are small. They are then transported inside the particle cloud in a region located in the range $x=1.5-2.5 \mathrm{~cm}$ where the large particle density is larger and the coagulation process is enhanced. In figure $16 \mathrm{~b}$ we represent the transport for $30 \mathrm{~nm}$ size particles inside the dust cloud. It appears that large particles are transported from the center of the cloud, i.e., $x \approx 1.8 \mathrm{~cm}$, to the periphery, i.e., $x \approx 1-1.5 \mathrm{~cm}$ and $x \approx 2-2.5 \mathrm{~cm}$. This shows that the production of larger particles is enhanced in the central zone of the dust cloud. It is worthy to mention here that the transport flux of larger particles is 1 order of magnitude slower than for the smallest one. This is due to the better confinement of the large particles that carry higher negative charges in the field reversal region. It is worthy to mention at this stage that the investigated discharge is generated in a closed vessel with no gas flow. Of course as shown in reference [13], the presence of gas flow may substantially affect the particle dynamic inside the discharge. 


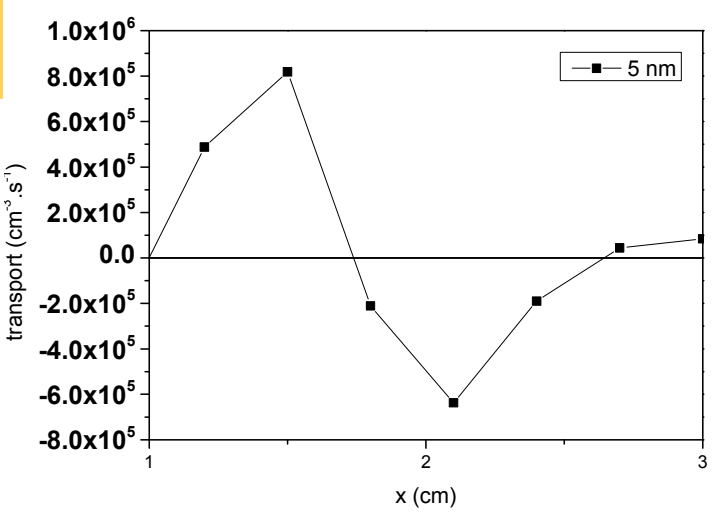

(a)

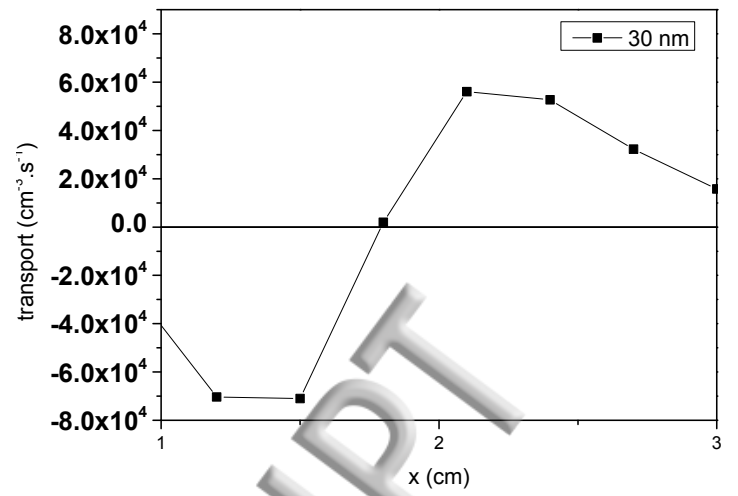

(b)

Figure 16: transport of particle of $5 \mathrm{~nm}$ (a) and $30 \mathrm{~nm}$ (b) particles in the dust cloud centered at 2 cm at $200 \mathrm{~s}$.

\section{Conclusion}

This study made possible understanding the detailed mechanism and dynamics of particles growth due to graphite cathode sputtering in an argon DC discharges. The modeling procedure described in this work enables us estimating and investigating the time- and space-evolution of the particle size distribution, and the corresponding total density, meandiameter and average-charge. Particle size distributions showed a bimodal behavior, with a minor, although very important, small particle population, a major core distribution and a strong population depletion for intermediate sizes. Both distribution modes are well represented by log-normal distribution. Comparison with experiment showed that the aerosol dynamic model yields a good prediction of the size-distribution behavior in the size range 10-100 $\mathrm{nm}$ where particle size could be measured. It also showed that the model also yields a good prediction of the total particle density. Sectional model yields therefore a much better prediction than a single size model for the particle density. The mean diameter values predicted using the sectional and the single-size distributions models are however very similar. Comparison with experiments showed that, although calculated and measured mean-diameter values are similar for long discharge durations, a significant difference on particle growth dynamics was observed.

The better particle density prediction obtained with the aerosol model is due to the more accurate description of the size- and charge- dependent coagulation growth process in this model. The sectional model makes it possible to properly describe the enhanced coagulation between particles with very different size. It also enables one taking into account the charge fluctuation that results in neutral or positively charged particle in the size range 1-5 nm. This charge fluctuation combined with relatively small charges are the driver for the coagulation process and govern the steady state particle density and the peculiar log-normal shape of the size distributions. These are characterized by a bimodal structure and a strong depletion in the size range 2-10 $\mathrm{nm}$, for long discharge durations. Analysis of the interplay between the 
different phenomena involved in the aerosol dynamics, shows that while the particle density is governed by coagulation, the mean diameter value is mainly determined by surface sticking process. This is the reason why aerosol dynamics and single size models yield the same prediction for the mean diameter value. The same analysis showed that the lifetime of small, 1-5 nm, particles is very short. These particles are consumed through coagulation with the large particle of the core distribution. This explains the strong depletion observed in the distribution. It also suggests a structure consisting of a collection of 1-5 nm particles embedded in a matrix that results from carbon deposition, for the large particles of the core distribution. This is fully consistent with the particle microstructures observed by SEM. We finally analyzed the space dynamics of the particle cloud. We observed that particle growth is located at the cloud center. The large particle obtained at this position diffuse to the cloud periphery. On the opposite the particle that nucleate in the cloud periphery are transported to the cloud center and supply at this position the small particle population that plays a keyrole in the coagulation process .

Of course, this model that deals with the description of quite complex plasmas can be substantially improved. In particular, we can take into account, in a self consistent way the coupling between cluster growth, i.e., particle nucleation, discharge equilibrium and aerosol dynamics. This would make it possible to describe additional effect such as the dusty plasma effect that appears at longer discharge durations and the coupling between the nucleation and the deposition processes on particle. Also, particle growth due to coagulation, in particular when involving large particles, may be further improved by taking into account enhancement effect such as dipole interaction between particle [16].

\section{References}

[1] G. I. Sukhinin, A. V. Fedoseev, S. N. Antipov et al., "Influence of Dust Particles Concentration on Plasma Parameters in DC Discharge," 10, 2009, pp. 781-785.

[2] B. M. Annaratone, and et al., "Agglomeration of mesoscopic particles in plasma," New Journal of Physics, vol. 11, no. 10, pp. 103013, 2009.

[3] A. Michau, G. Lombardi, L. Colina Delacqua et al., "Field Reversal and Particle Growth in DC Discharge," Plasma Chemistry and Plasma Processing, pp. 1-20, 2012.

[4] A. Michau, C. Arnas, G. Lombardi et al., "Nanoparticle formation and dusty plasma effects in DC sputtering discharge with graphite cathode," Plasma Sources Science and Technology, vol. 25, no. 1, pp. 015019, 2016.

[5] S. Friedlander, Smoke, dust and haze : fundamentals of aerosol dynamics: Oxford University Press, 2000. 
This manuscript was accepted by J. Appl. Phys. Click here to see the version of record.

[6] J. Berndt, E. Kovačević, I. Stefanović et al., "Some Aspects of Reactive Complex Plasmas," Contributions to Plasma Physics, vol. 49, no. 3, pp. 107-133, 2009.

[7] C. Arnas, A. Michau, G. Lombardi et al., "Effects of the growth and the charge of carbon nanoparticles on direct current discharges," Physics of Plasmas, vol. 20, no. 1, Jan, 2013.

[8] C. Dominique, and C. Arnas, "Cathode sputtering and the resulting formation of carbon nanometer-size dust," Journal of Applied Physics, vol. 101, no. 12, pp. 123304-8, 2007.

[9] E. Oyarzabal, R. P. Doerner, M. Shimada et al., "Carbon atom and cluster sputtering under low-energy noble gas plasma bombardment," Journal of Applied Physics, vol. 104, no. 4, pp. 043305-12, 2008.

[10] A. Michau, G. Lombardi, C. Arnas et al., "Modeling of dust formation in a DC discharge," Journal of Nuclear Materials, vol. 415, no. 1, Supplement, pp. S1077-S1080, 2011.

[11] A. Michau, K. Hassouni, C. Arnas et al., "On the Competition Between the Phenomena Involved in the Aerosol Dynamics in Sputtering Nonequilibrium Plasma," IEEE Transactions on Plasma Science, vol. 44, no. 4, pp. 528-534, 2016.

[12] J. Goree, "Charging of particles in a plasma," Plasma Sources Science and Technology, vol. 3, no. 3, pp. 400-406, 1994.

[13] P. Agarwal, and S. L. Girshick, "Sectional modeling of nanoparticle size and charge distributions in dusty plasmas," Plasma Sources Science and Technology, vol. 21, no. 5, pp. 055023, 2012.

[14] D.-J. Kim, and K.-S. Kim, "Analysis on nanoparticle growth by coagulation in silane plasma reactor," AlChE journal, vol. 48, no. 11, pp. 2499-2509, 2002.

[15] T. Matsoukas, and M. Russell, "Particle charging in low-pressure plasmas," Journal of Applied Physics, vol. 77, no. 9, pp. 4285-4292, 1995.

[16] L. Ravi, and S. L. Girshick, "Coagulation of nanoparticles in a plasma," Physical Review E, vol. 79, no. 2, pp. 026408, 2009.

[17] V. I. Kolobov, and L. D. Tsendin, "Analytic model of the cathode region of a short glow discharge in light gases," Physical Review A, vol. 46, no. 12, pp. 7837, 1992.

[18] M. A. Lieberman, and A. J. Lichtenberg, Principles of plasma discharges and materials processing, New York: John Wiley \& Sons, 1994.

[19] A. Michau, G. Lombardi, L. Colina Delacqua et al., "Modeling carbonaceous particle formation in an argon graphite cathode dc discharge," Plasma Physics and Controlled Fusion, vol. 52, no. 12, pp. 124014, 2010.

[20] F. Gelbard, Y. Tambour, and J. H. Seinfeld, "Sectional representations for simulating aerosol dynamics," Journal of Colloid and Interface Science, vol. 76, no. 2, pp. 541-556, 1980.

[21] D. R. Warren, and J. H. Seinfeld, "Simulation of Aerosol Size Distribution Evolution in Systems with Simultaneous Nucleation, Condensation, and Coagulation," Aerosol Science and Technology, vol. 4, no. 1, pp. 31-43, 1985.

[22] U. Kortshagen, and U. Bhandarkar, "Modeling of particulate coagulation in low pressure plasmas," Physical Review E, vol. 60, no. 1, pp. 887, 1999.

[23] D. J. Kim, and K. S. Kim, "Analysis on nanoparticle growth by coagulation in silane plasma reactor," AIChE journal, vol. 48, no. 11, pp. 2499-2509, 2002.

[24] U. Bhandarkar, U. Kortshagen, and S. Girshick, "Numerical study of the effect of gas temperature on the time for onset of particle nucleation in argon silane low-pressure plasmas," Journal of Physics D: Applied Physics, vol. 36, no. 12, pp. 1399, 2003.

[25] K. De Bleecker, A. Bogaerts, and W. Goedheer, "Modelling of nanoparticle coagulation and transport dynamics in dusty silane discharges," New Journal of Physics, vol. 8, no. 9, pp. 178, 2006.

[26] S. Warthesen, and S. Girshick, "Numerical simulation of the spatiotemporal evolution of a nanoparticle-plasma system," Plasma Chemistry and Plasma Processing, vol. 27, no. 3, pp. 292-310, 2007. 
This manuscript was accepted by J. Appl. Phys. Click here to see the version of record.

[27] L. Couëdel, A. Samarian, M. Mikikian et al., "Influence of the ambipolar-to-free diffusion transition on dust particle charge in a complex plasma afterglow," Physics of Plasmas, vol. 15, no. 6, pp. 063705, 2008.

[28] K. De Bleecker, A. Bogaerts, and W. Goedheer, "Modeling of the formation and transport of nanoparticles in silane plasmas," Physical Review E, vol. 70, no. 5, pp. 056407, 2004.

[29] C. A. A. Zeinert, C. Dominique, and A. Mouberi "Optical properties of carbonaceous nanoparticles produced in sputtering discharges," Journal of Vacuum Science \& Technology A: Vacuum, Surfaces, and Films, vol. 26, no. 6, pp. 1450-1454, 2008/11/01, 2008.

[30] C. Arnas, A. Mouberi, K. Hassouni et al., "Carbon dust formation in a cold plasma from cathode sputtering," Journal of Nuclear Materials, vol. 390-391, pp. 140-143, 2009.

[31] K. Kumar K, L. Couëdel, and C. Arnas, "Nanoparticles in direct-current discharges: Growth and electrostatic coupling," Journal of Plasma Physics, vol. 80, no. 6, pp. 849-854, 2014/12/001, 2014. 


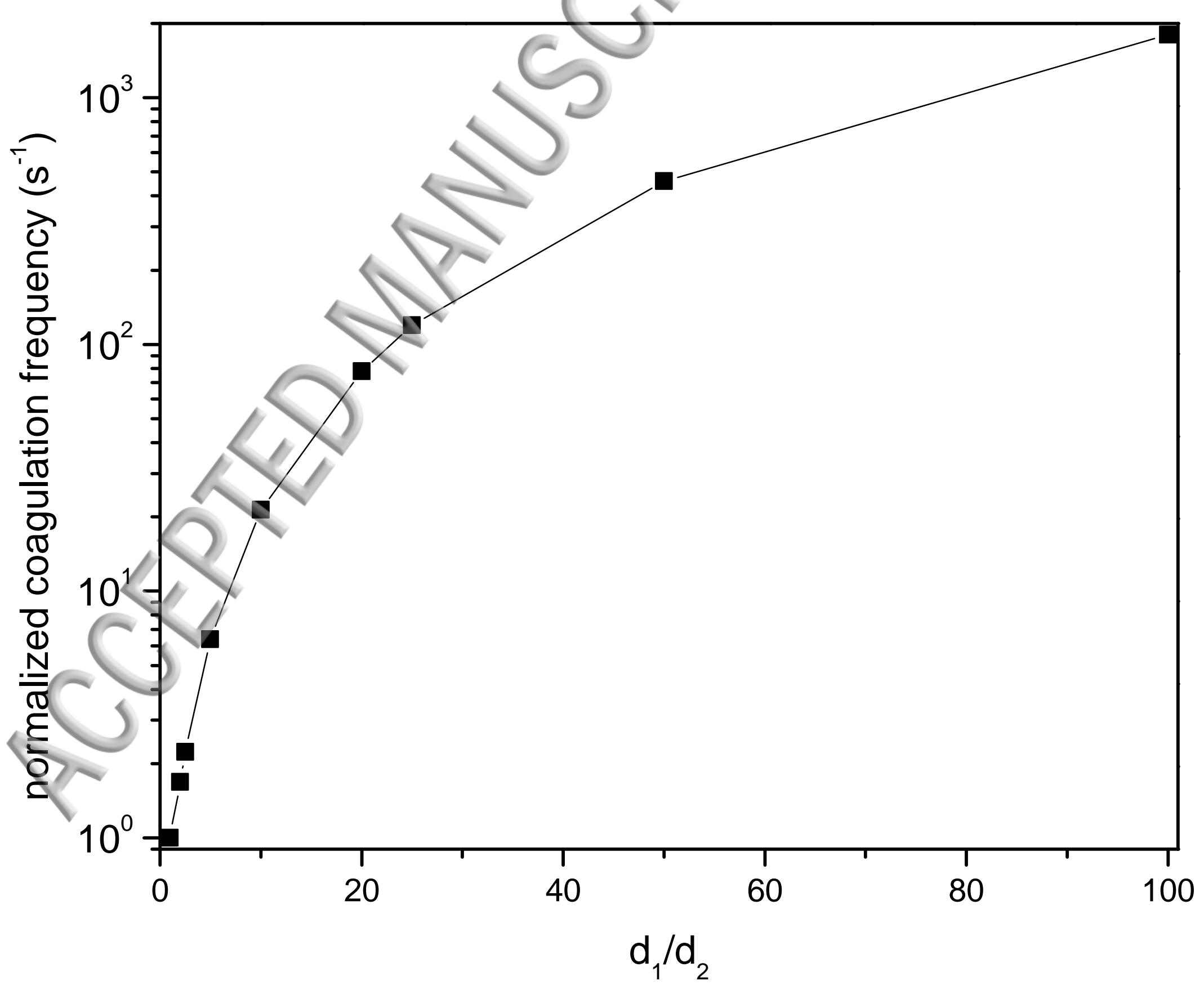




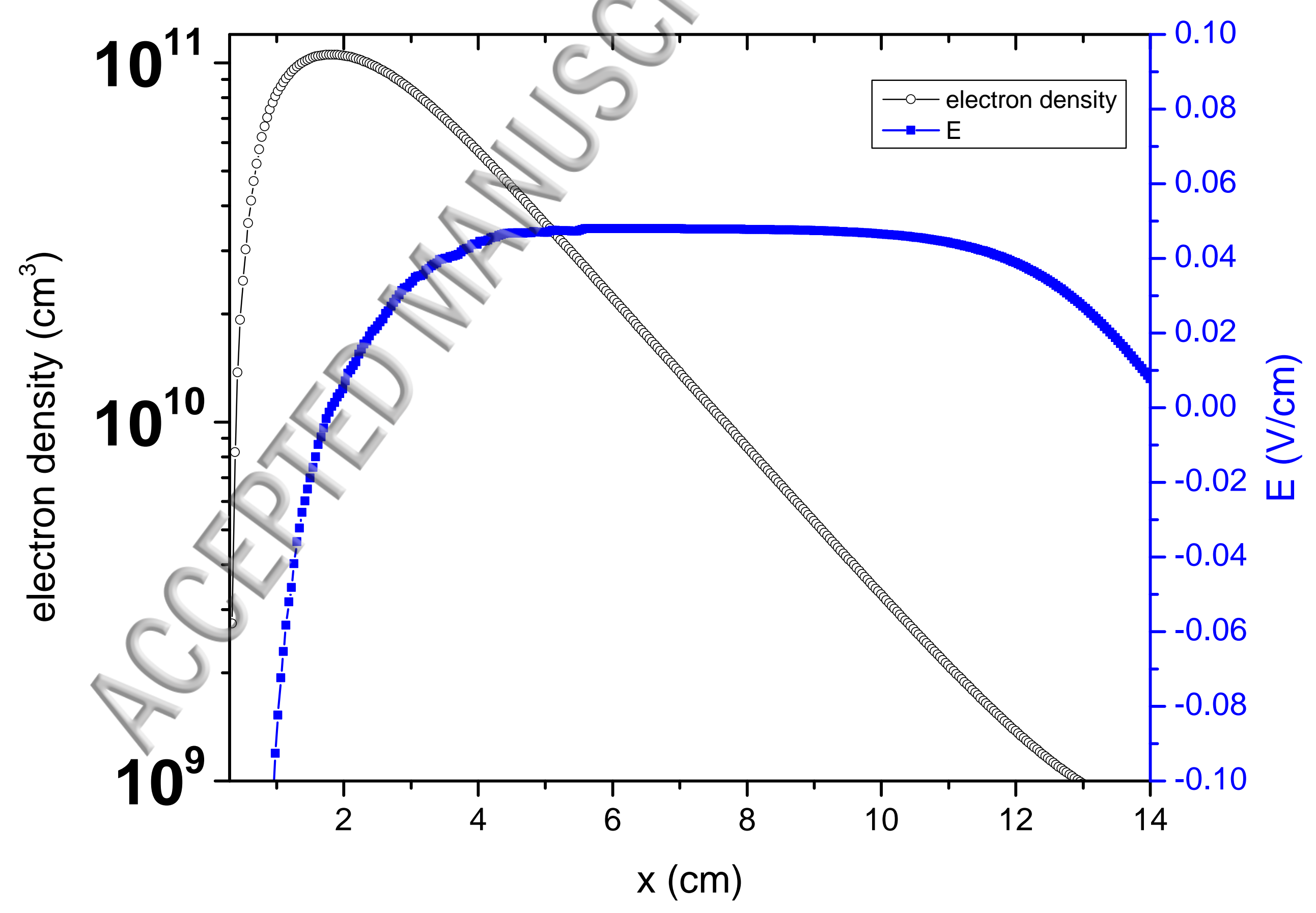




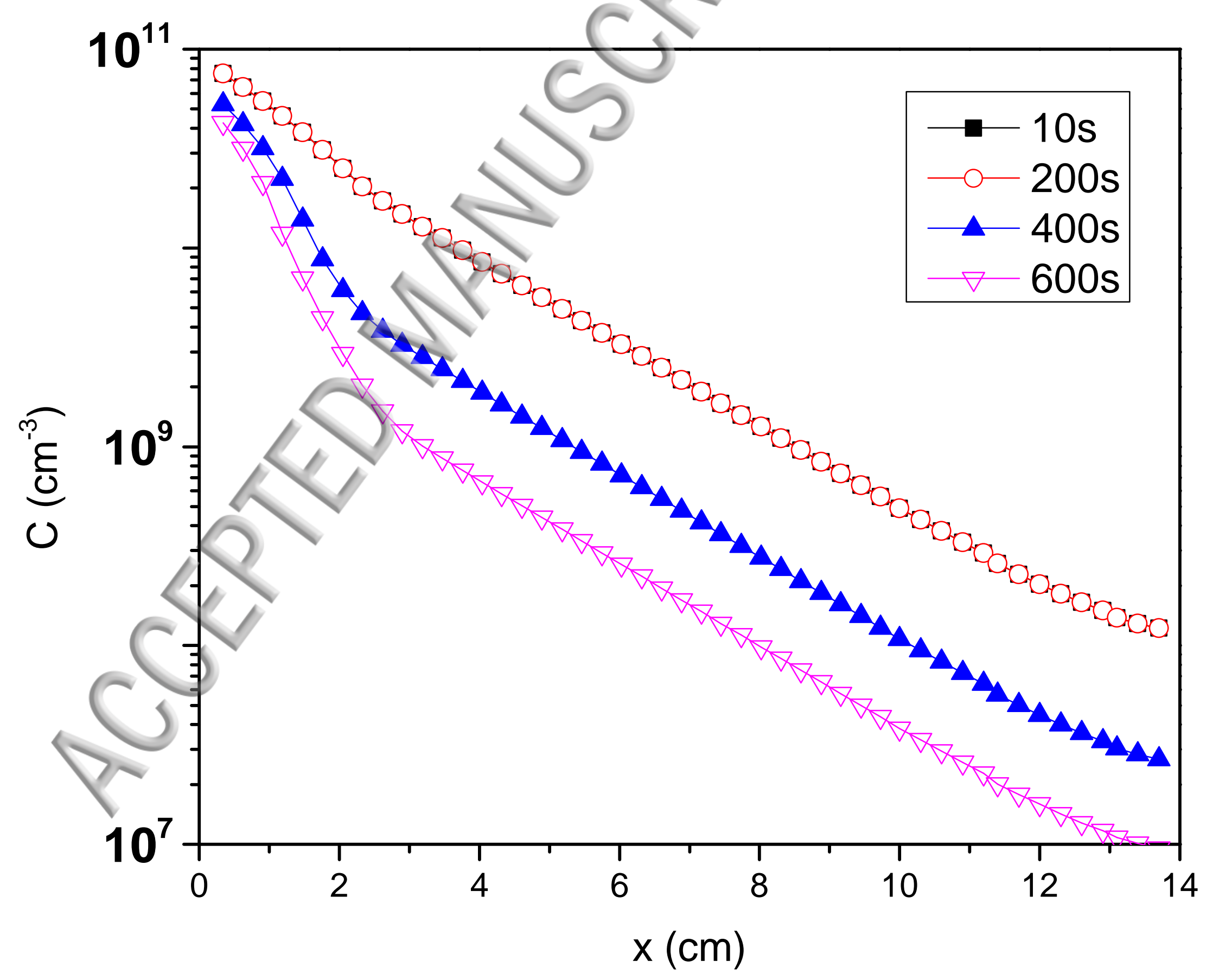




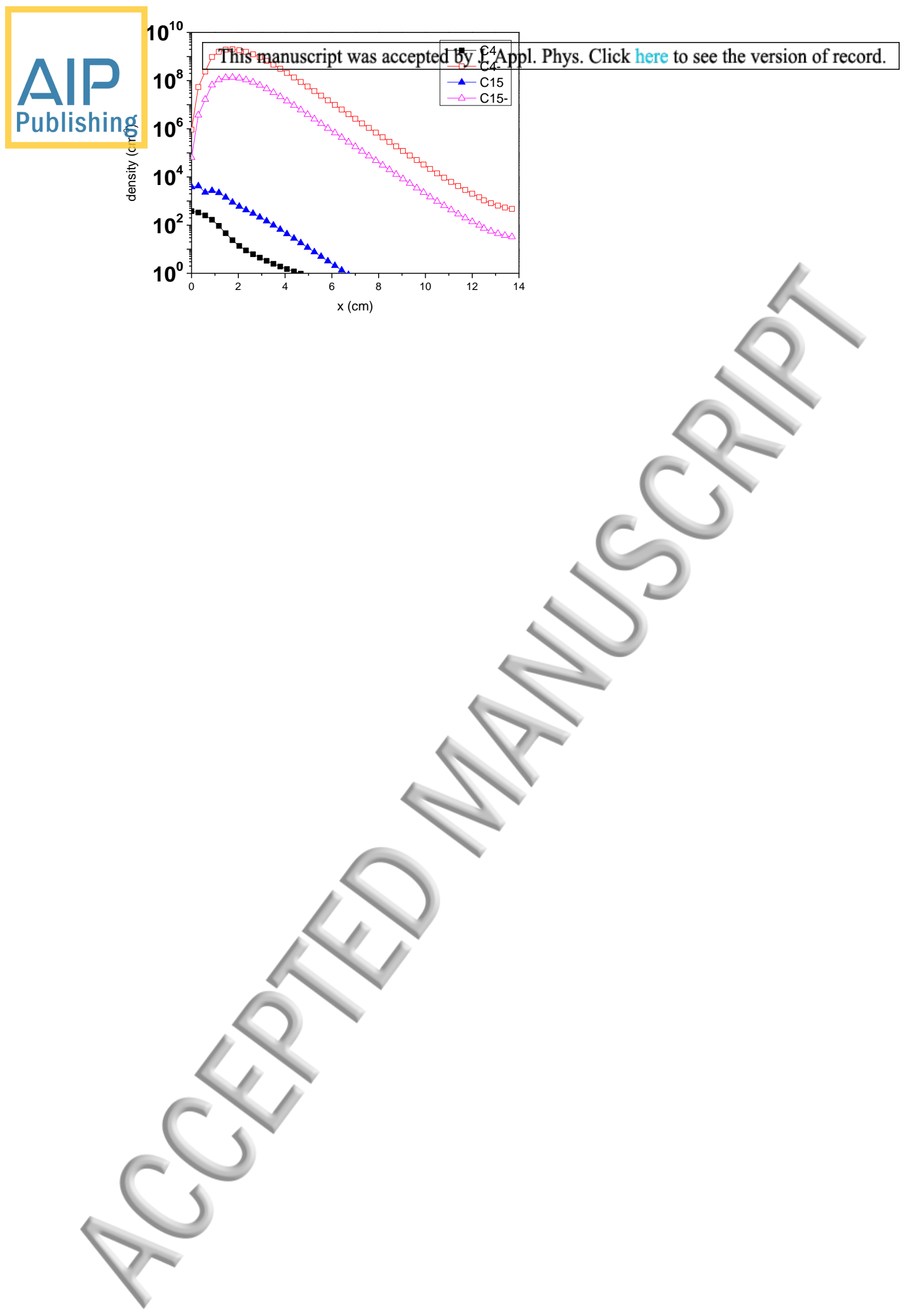




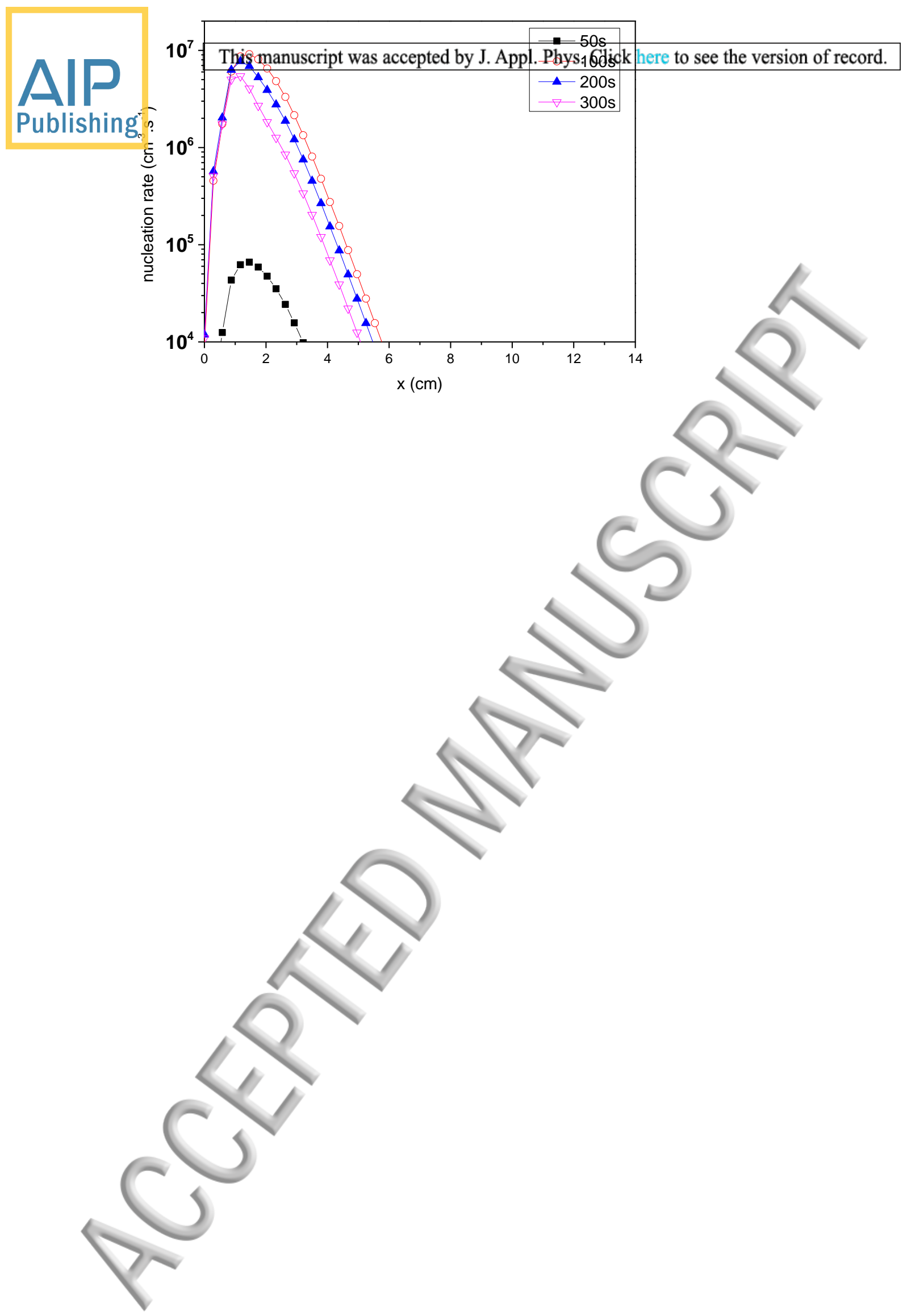




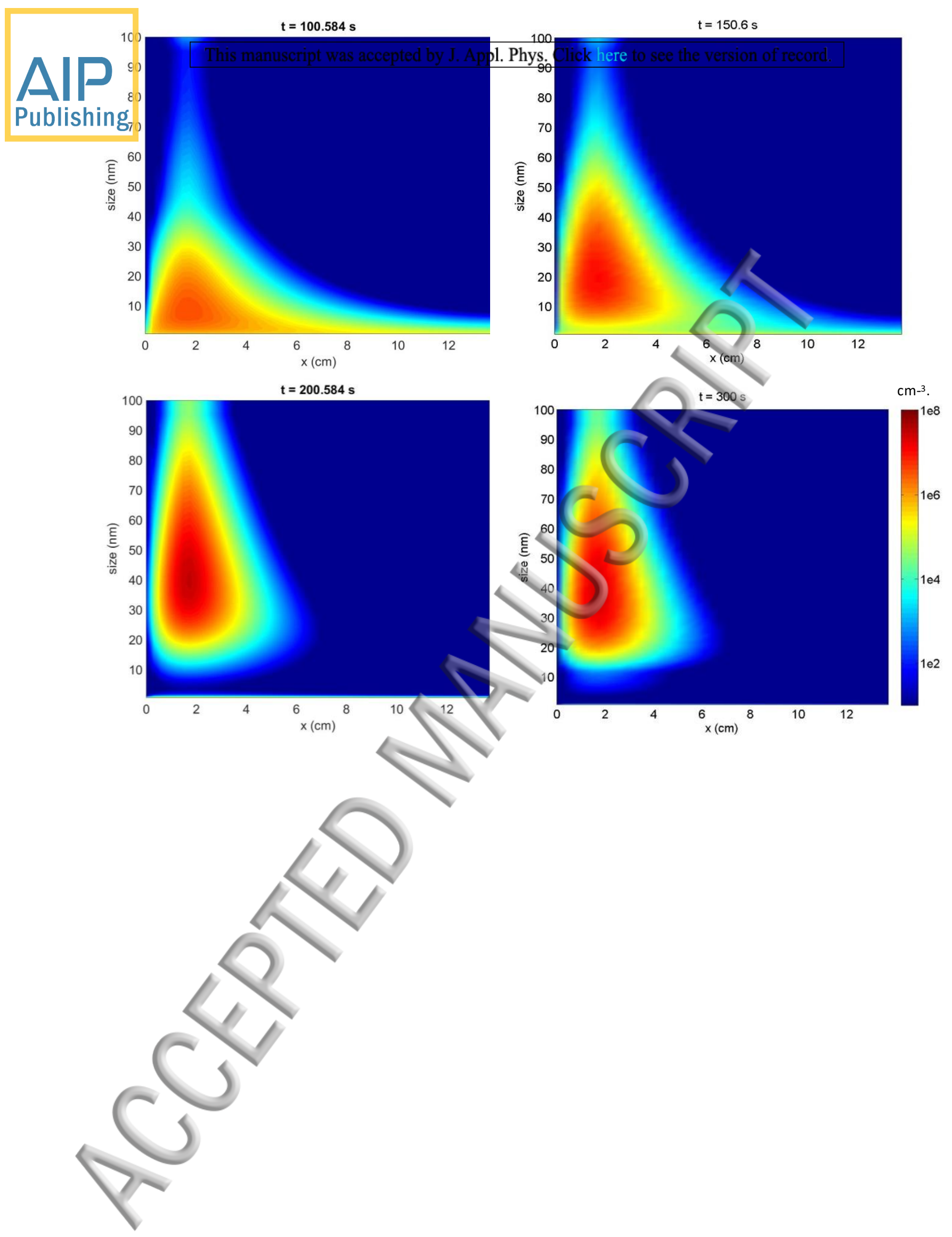




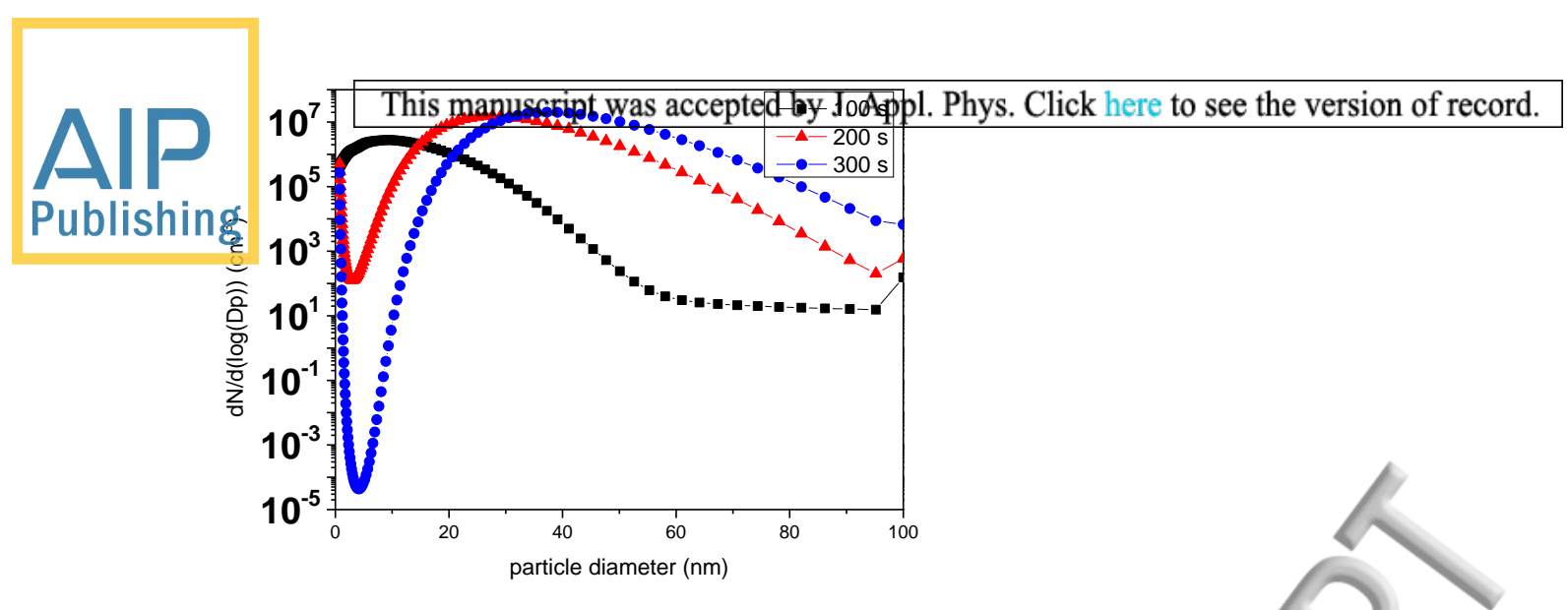




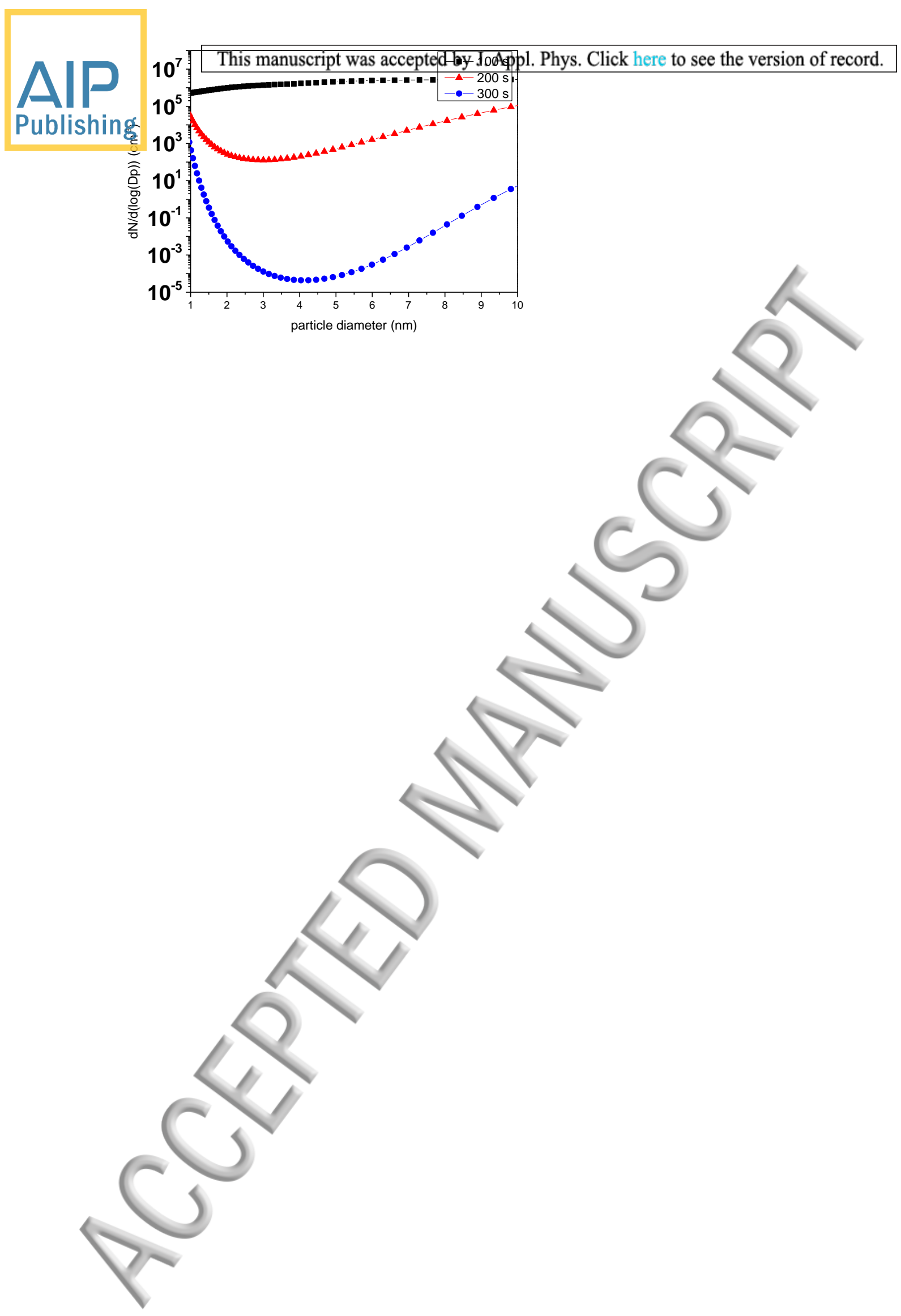




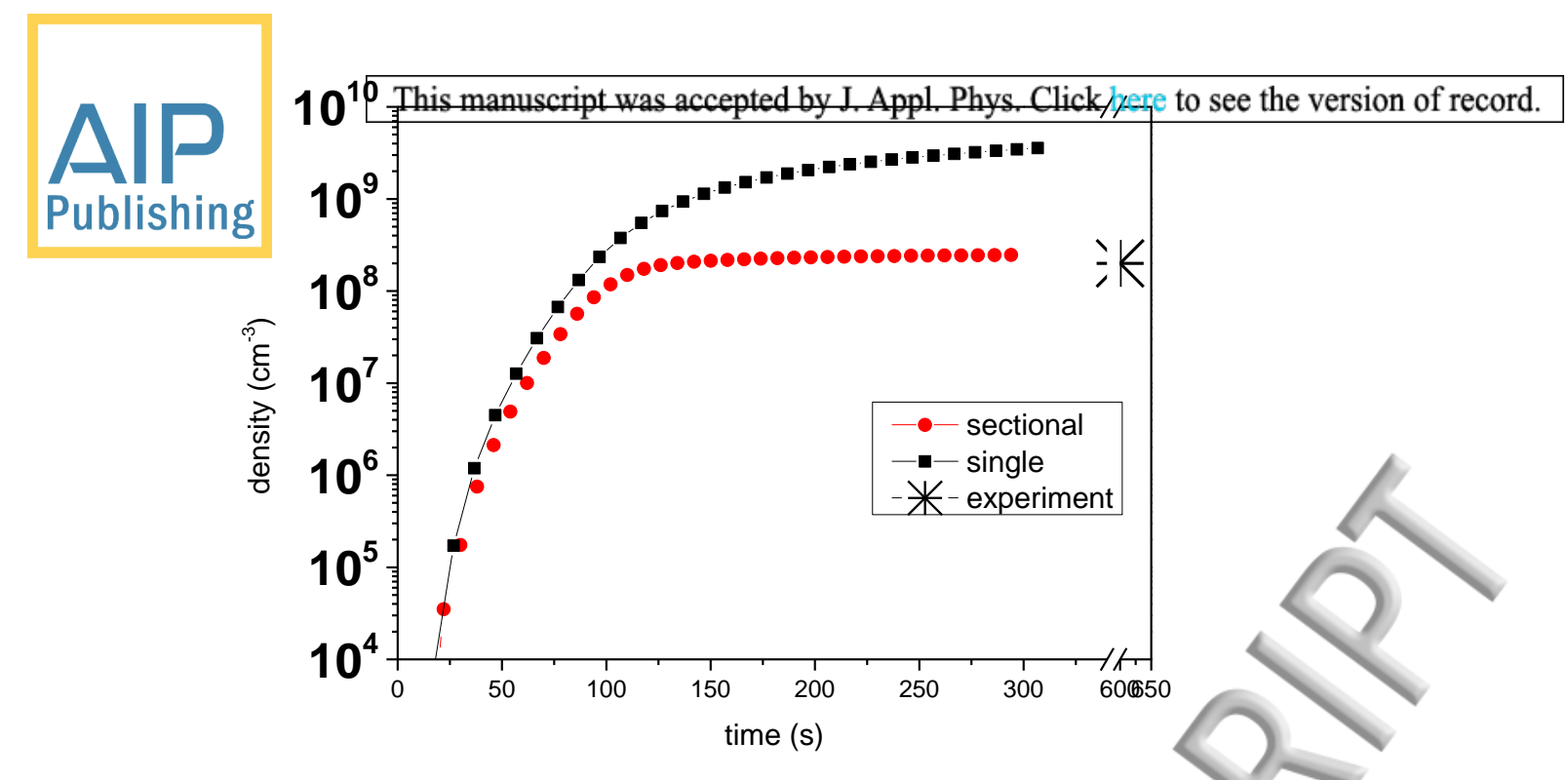



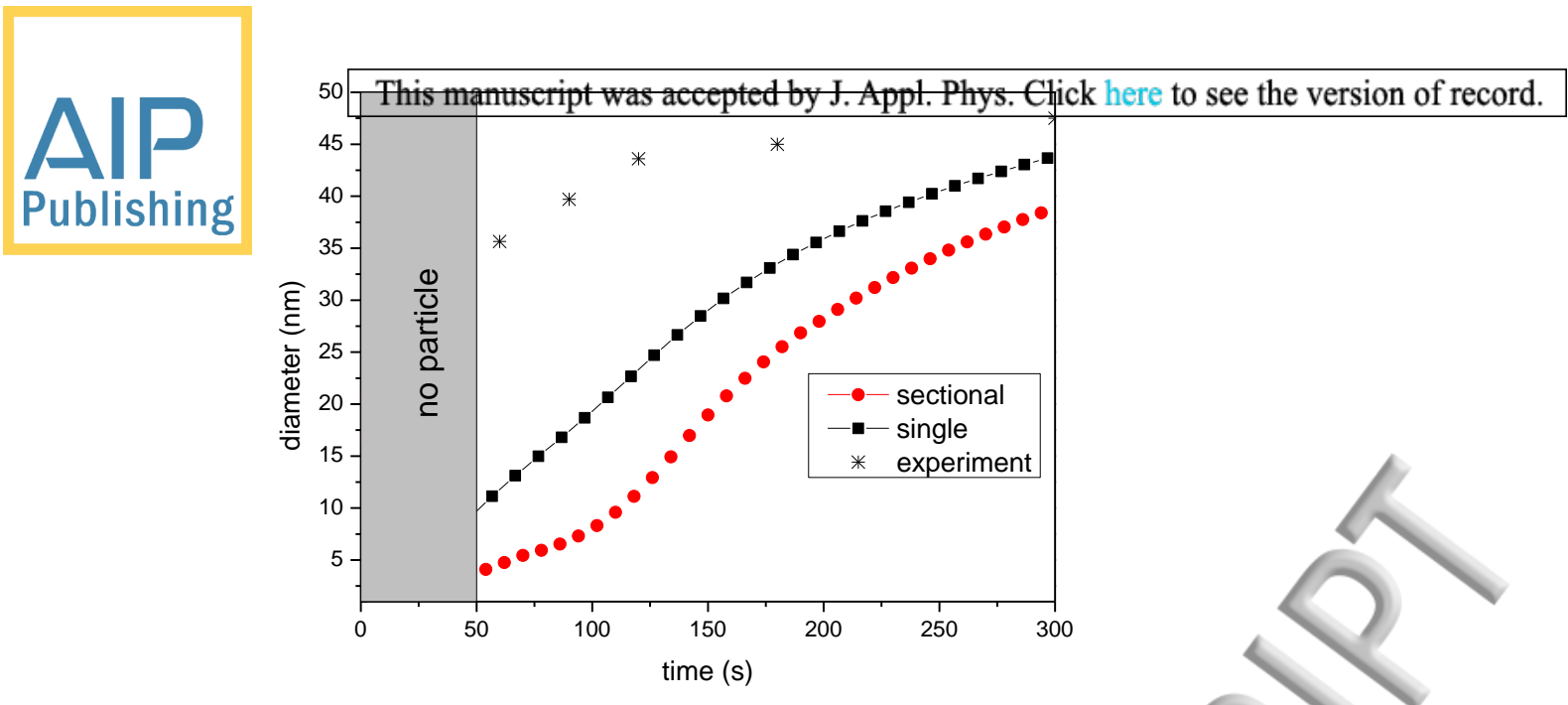


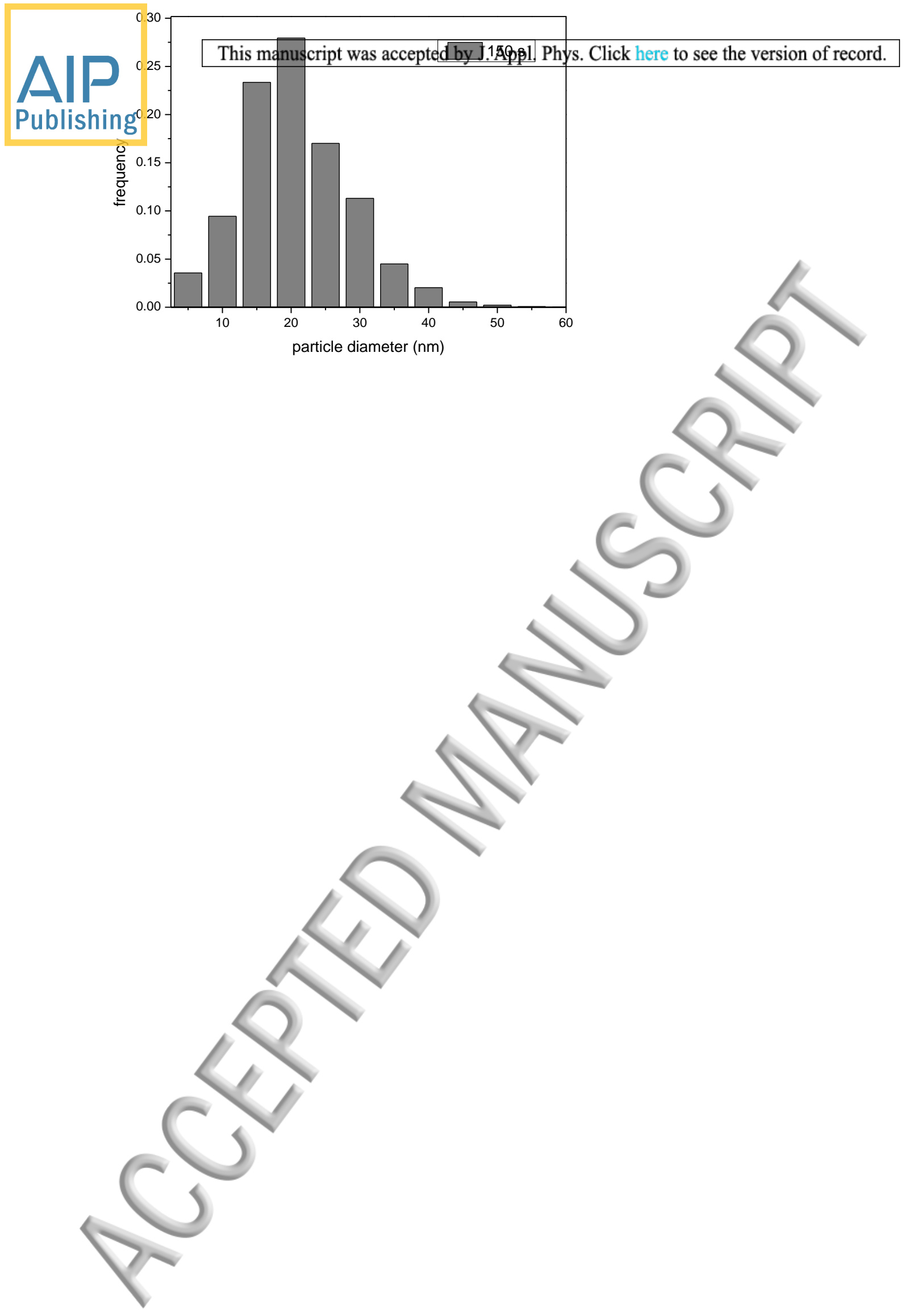




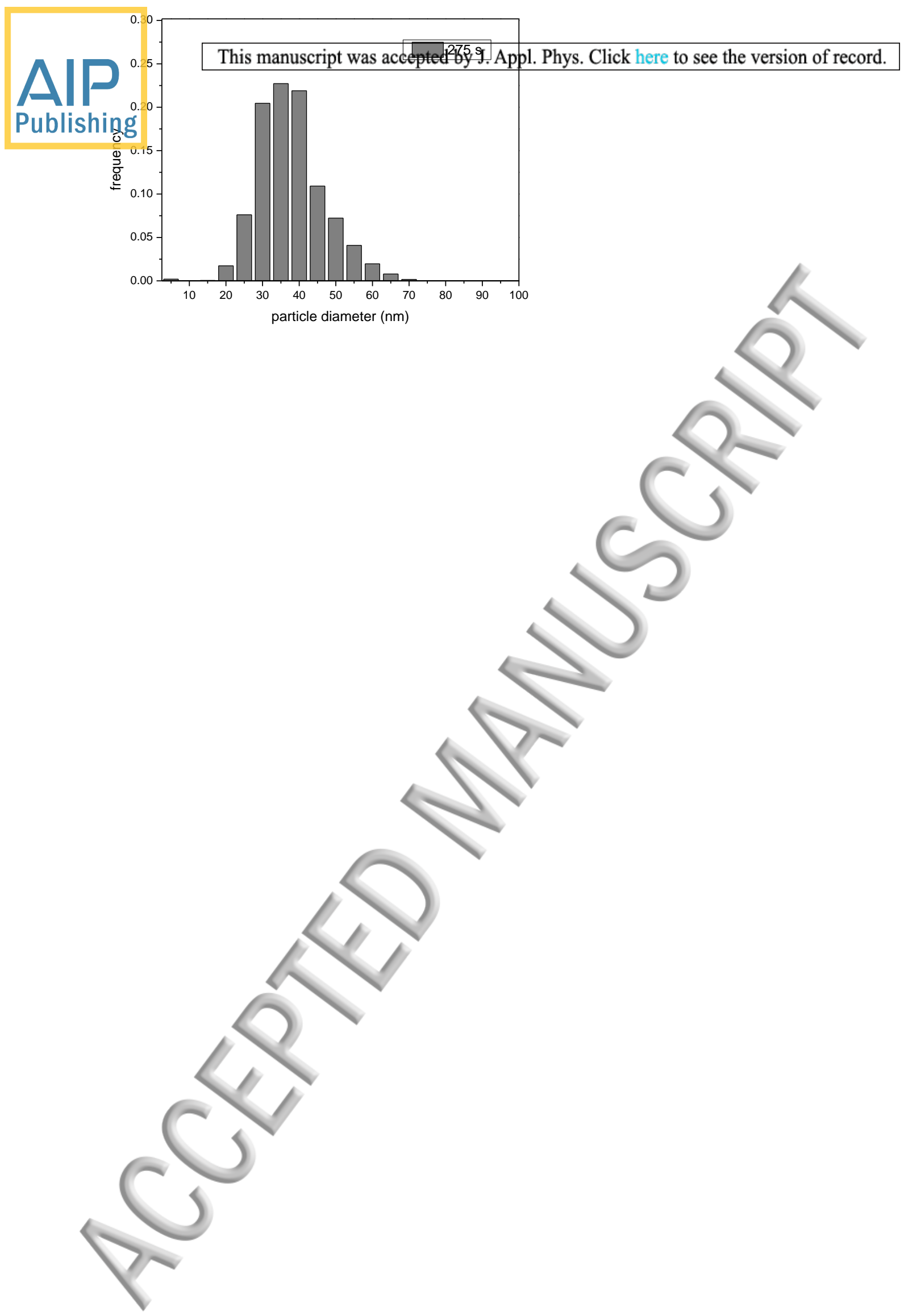




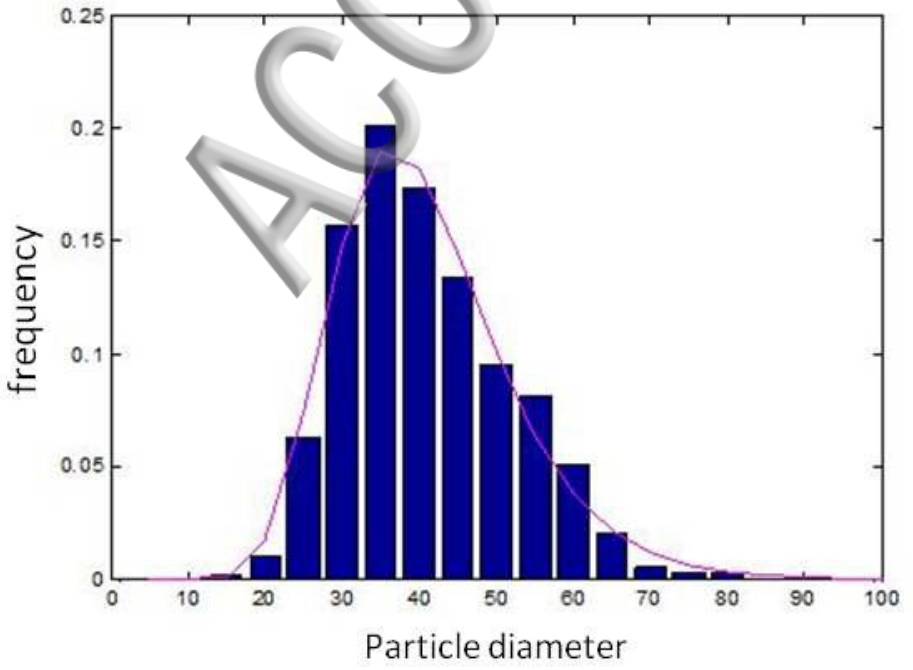




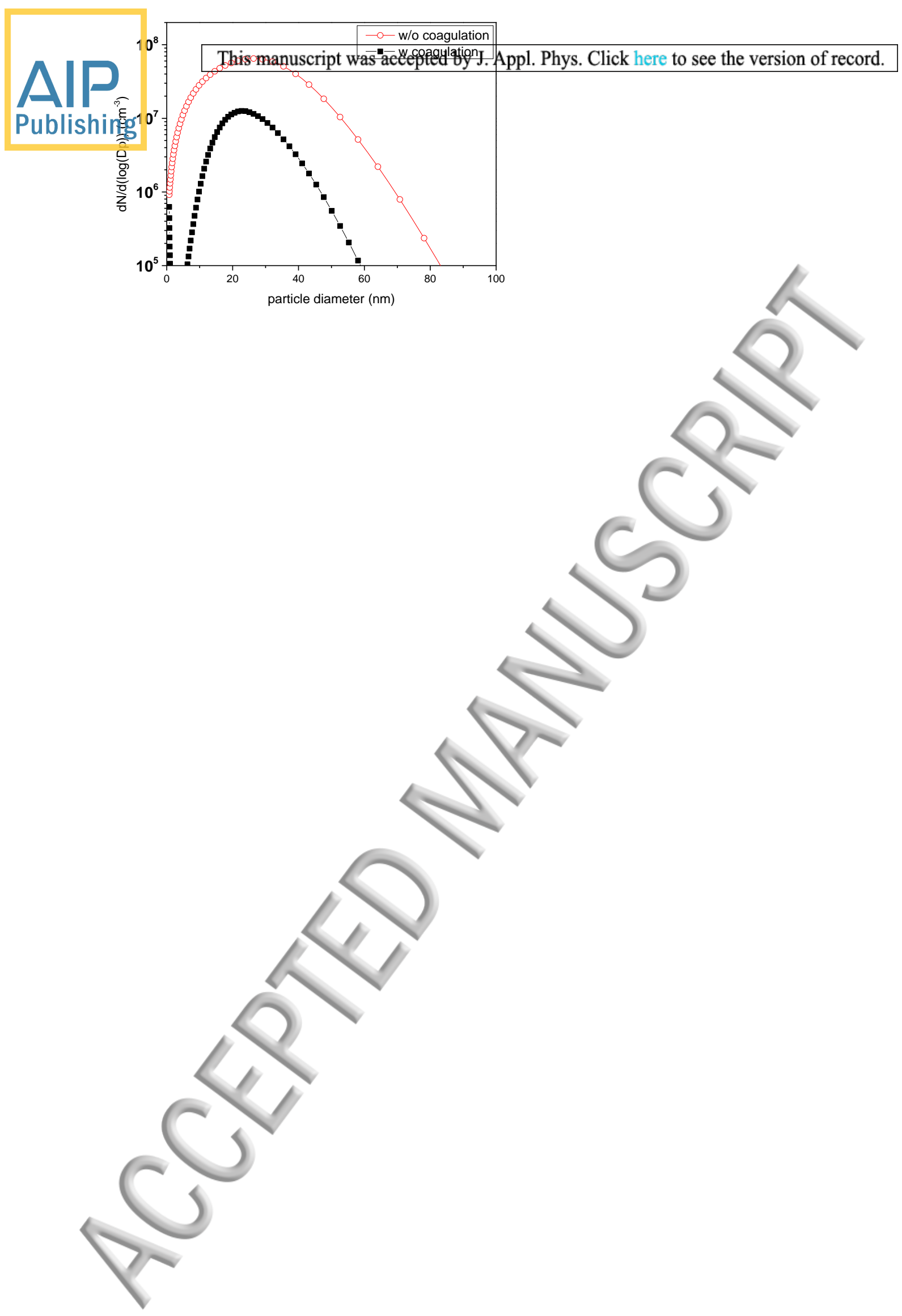




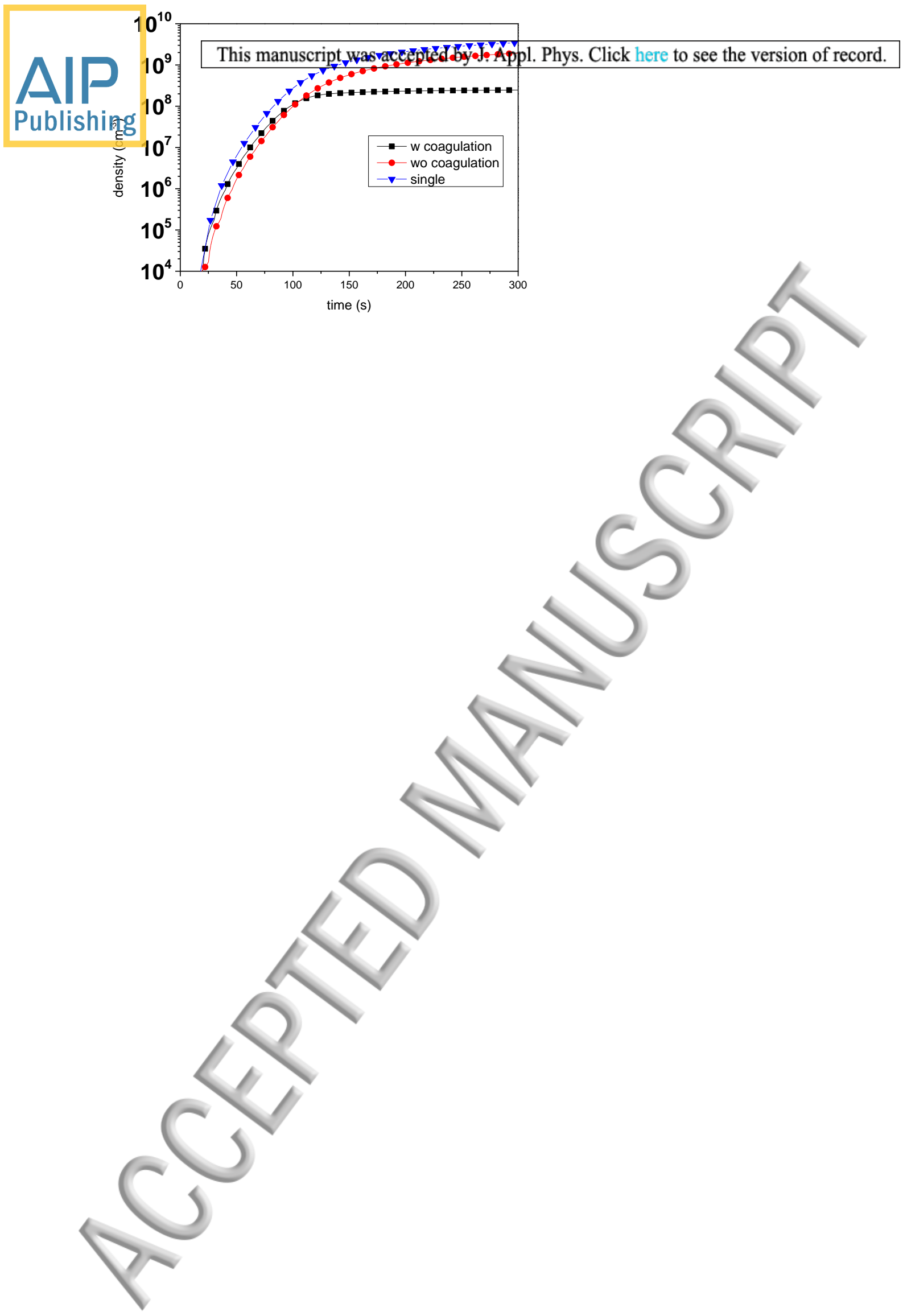




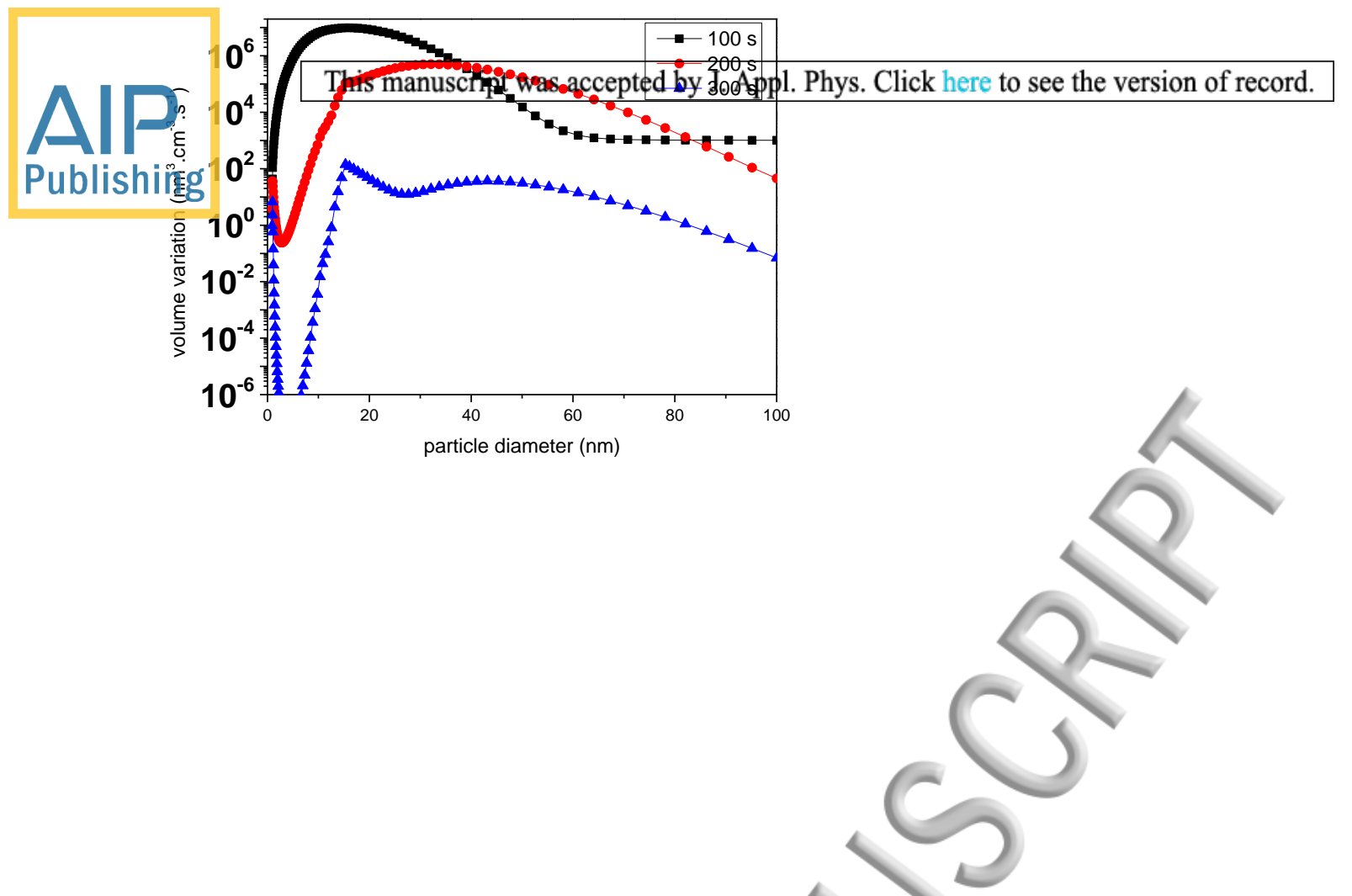




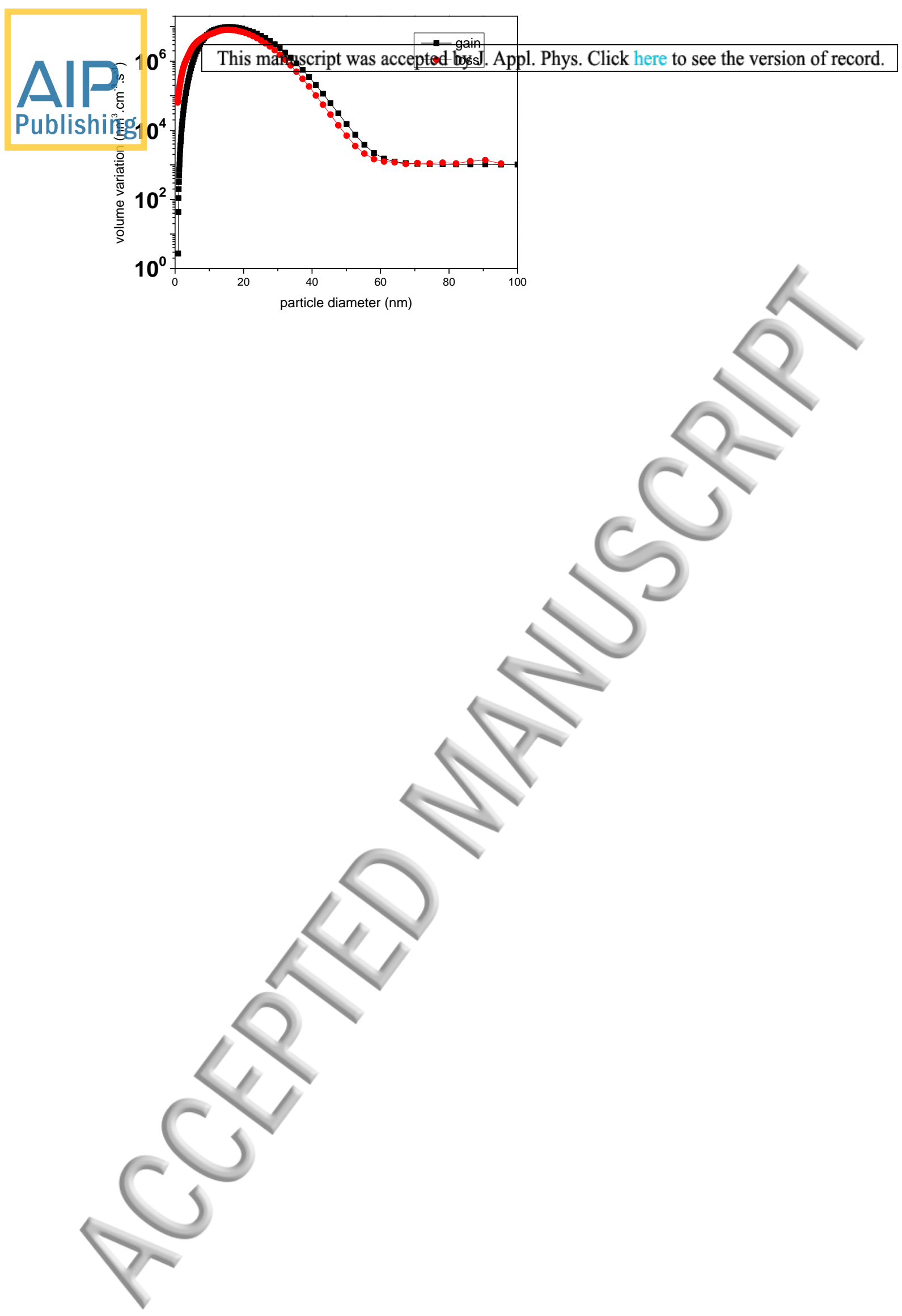




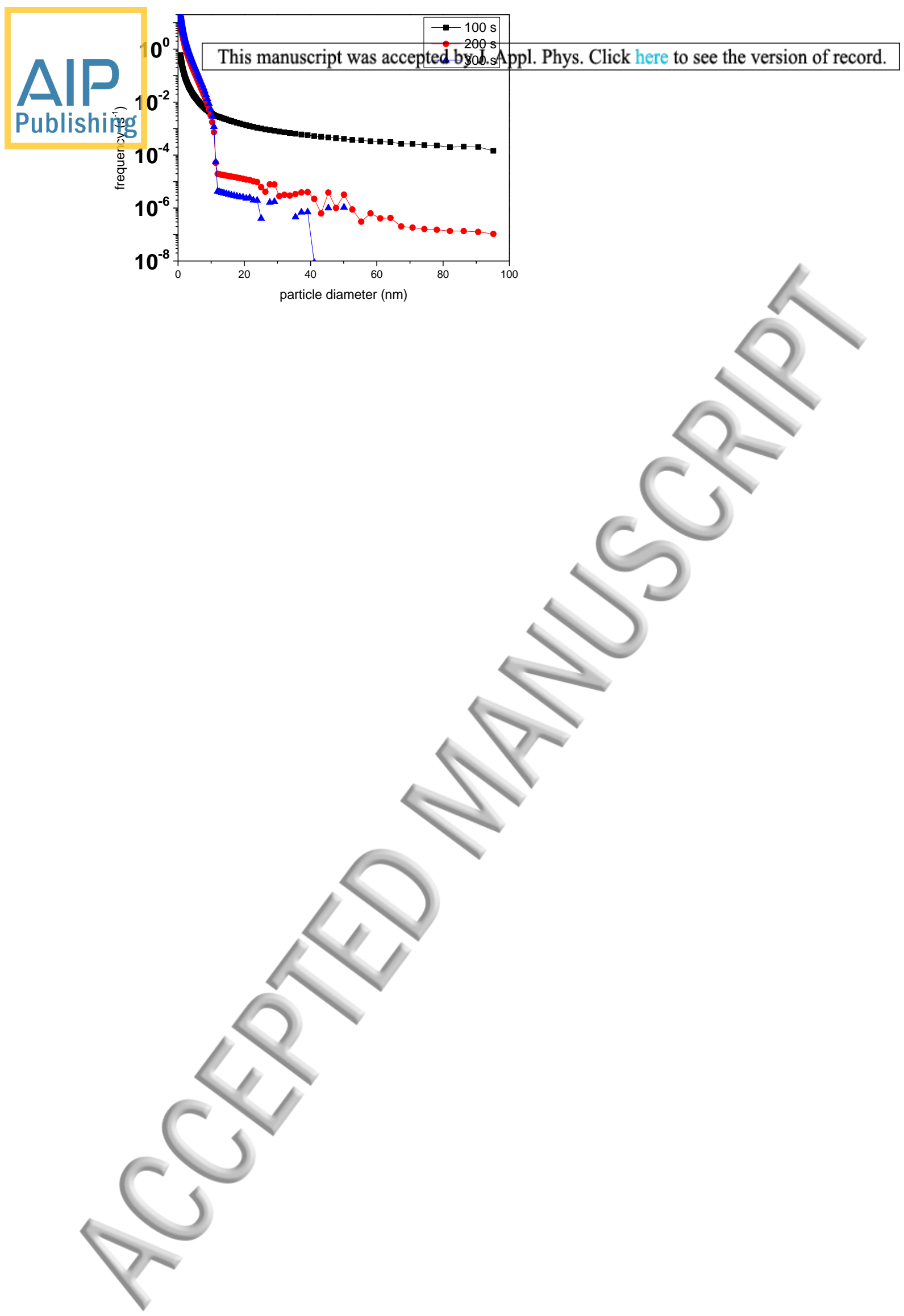




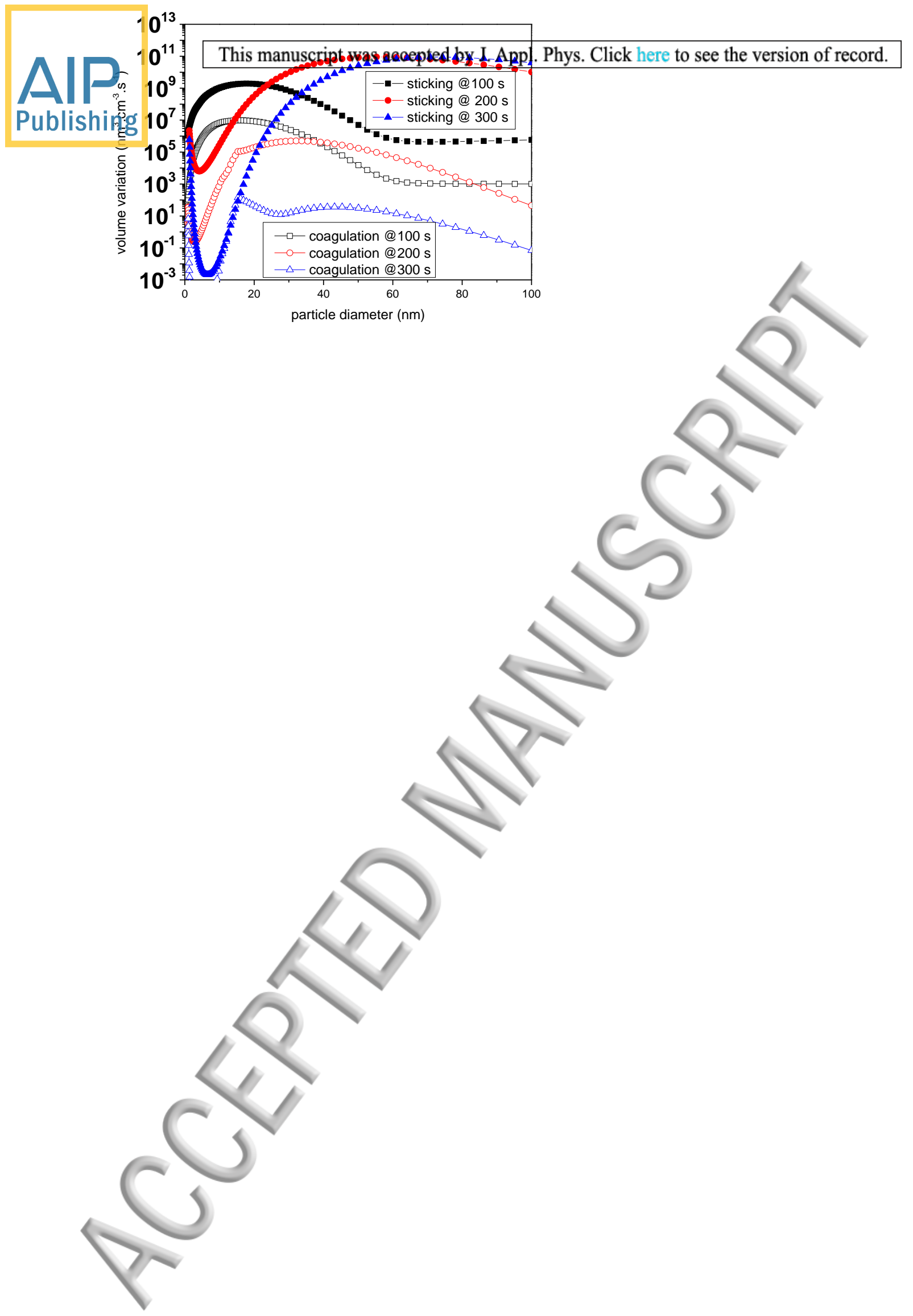




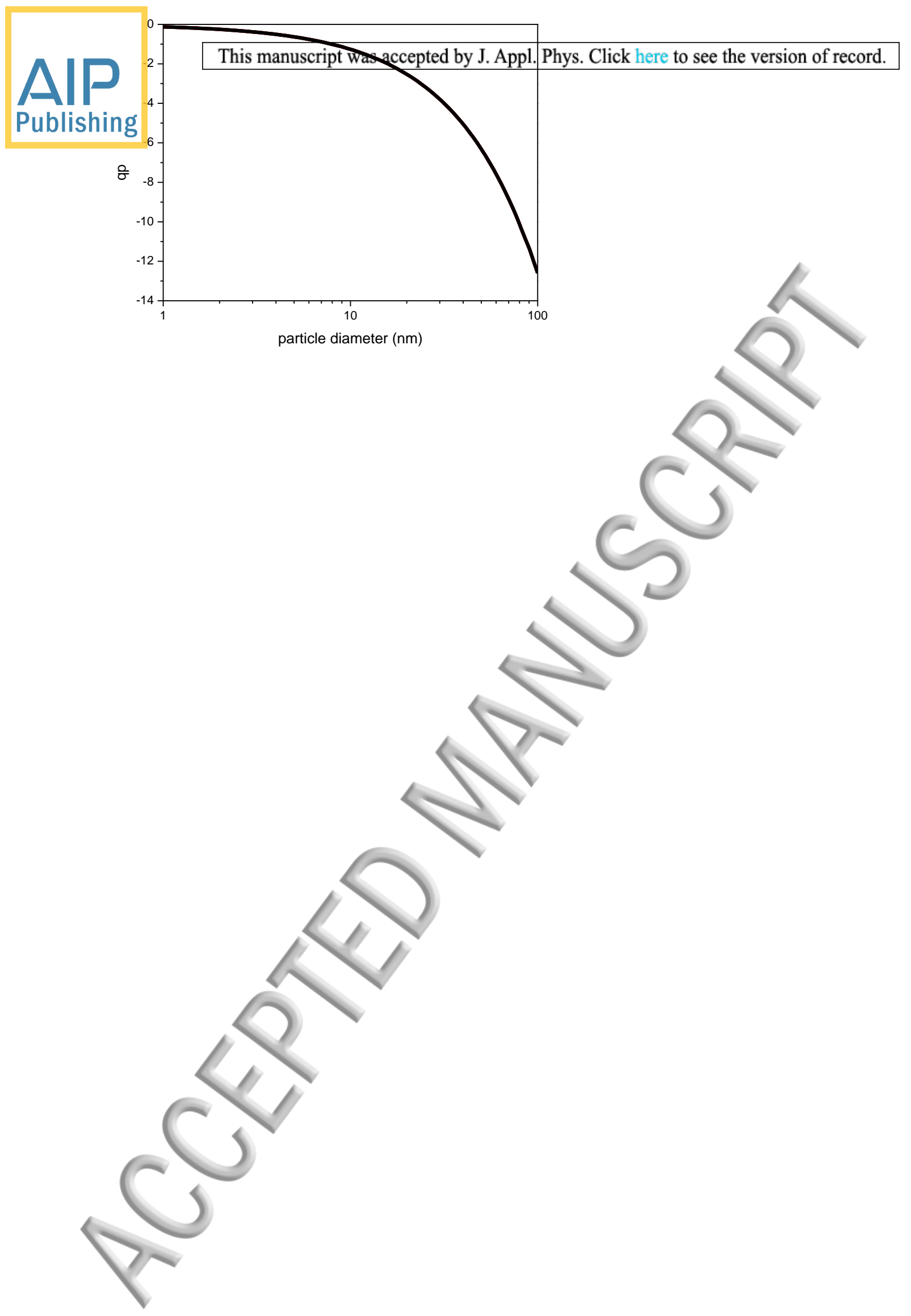




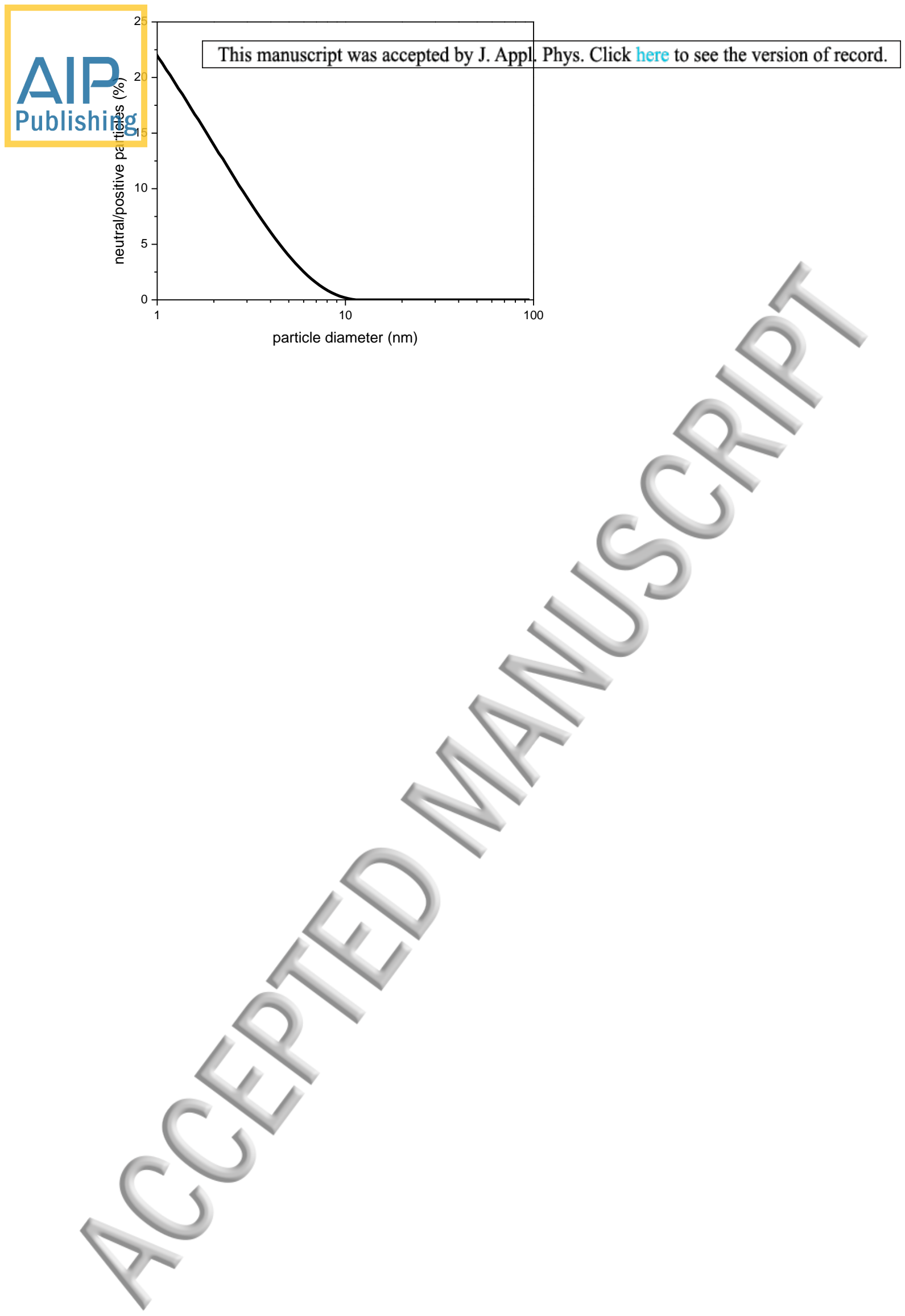




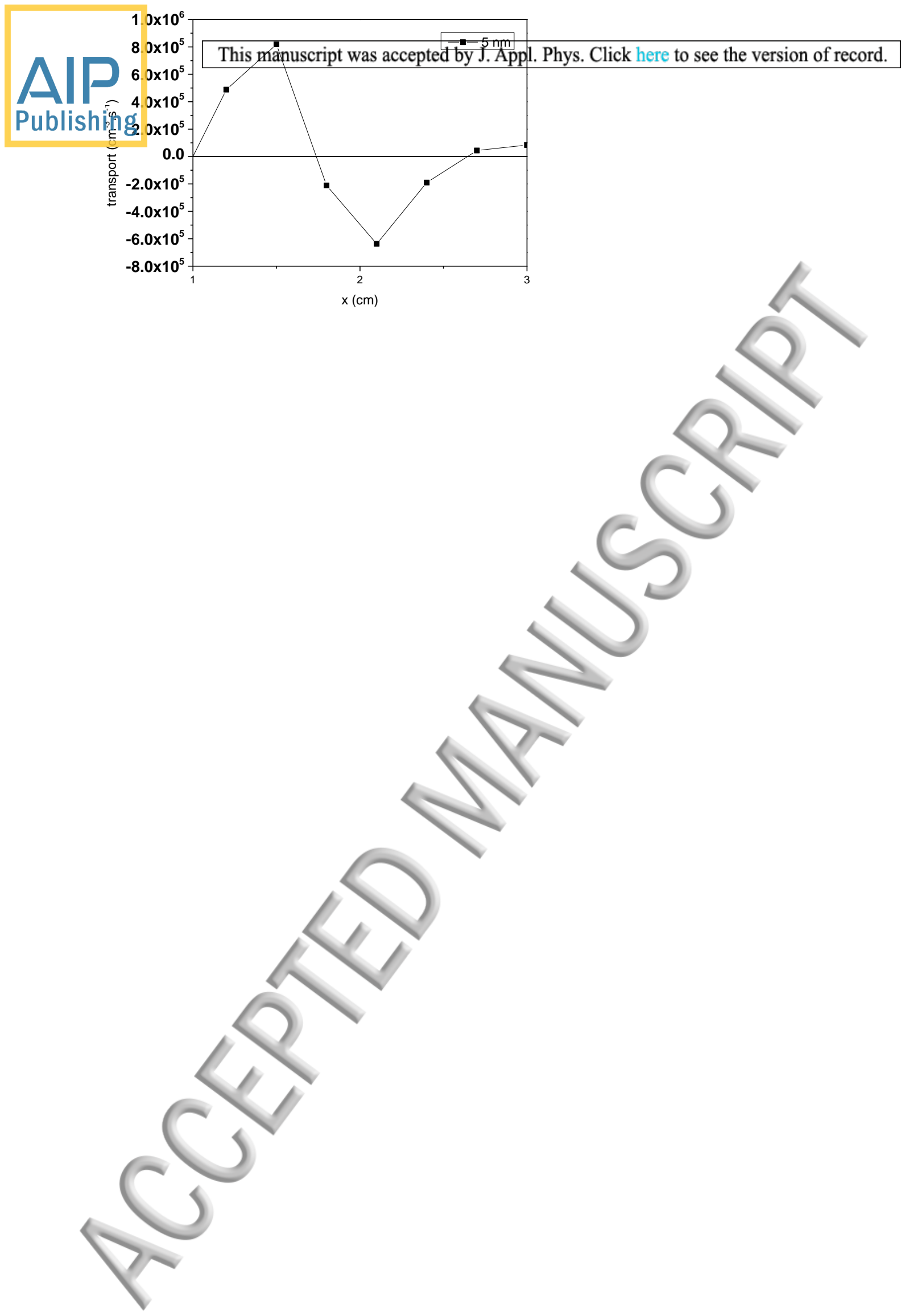




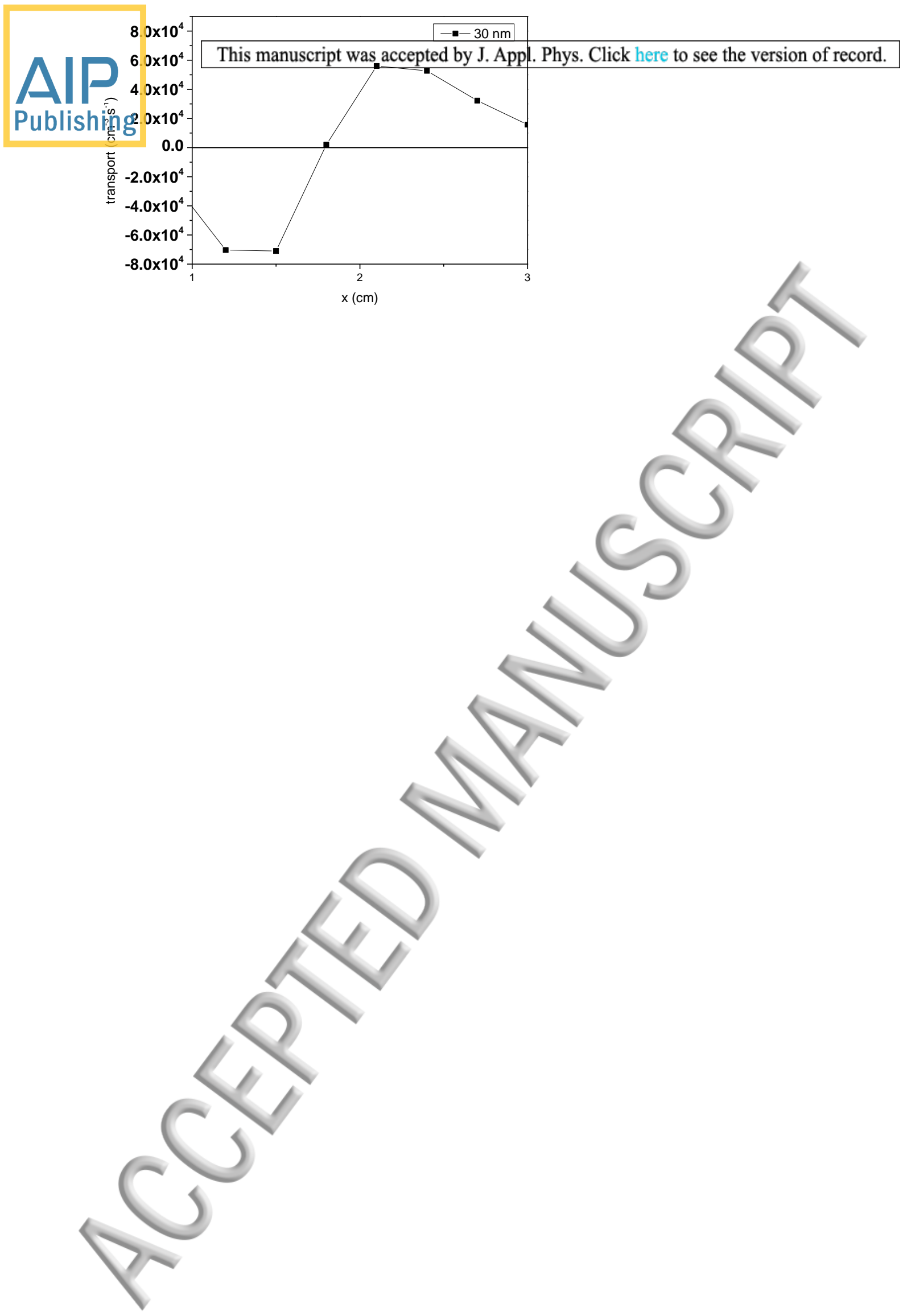

